\title{
Transcriptome-wide and Expression Analysis of the NAC Gene family in Pepino (Solanum muricatum) during drought stress
}

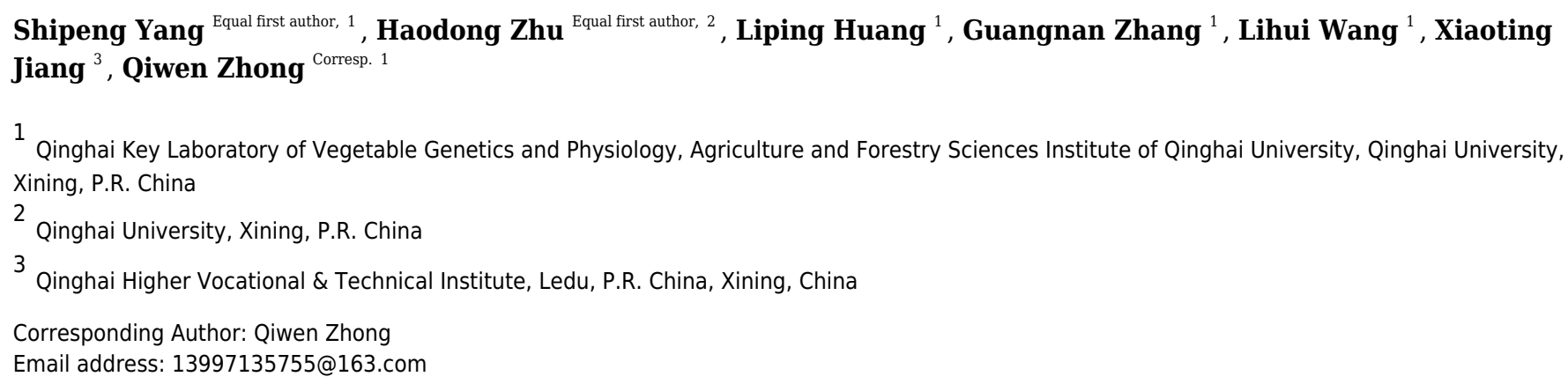

Solanum muricatum (Pepino) is an increasingly popular solanaceous crop and is tolerant of drought conditions. In this study, 71 NAC transcription factor family genes of Solanum muricatum were selected to provide a theoretical basis for subsequent in-depth study of their regulatory roles in the response to biological and abiotic stresses, and were subjected to whole-genome analysis. The NAC sequences obtained by transcriptome sequencing were subjected to bioinformatics prediction and analysis. Three concentration gradient drought stresses were applied to the plants, and the target gene sequences were analyzed by qPCR to determine their expression under drought stress. The results showed that the Solanum muricatum NAC family of contains 71 genes, 47 of which have conserved domains. The protein sequence length, molecular weight, hydrophilicity, aliphatic index, and isoelectric point of these transcription factors were predicted and analyzed.

Phylogenetic analysis showed that the Solanum muricatum NAC gene family is divided into seven subfamilies. Some NAC genes of Solanum muricatum are closely related to the NAC genes of Solanaceae crops such as tomato, pepper, and potato. The seedlings of Solanum muricatum were grown under different gradients of drought stress conditions and qPCR was used to analyze the NAC expression in roots, stems, leaves, and flowers. The results showed that 13 genes did not respond to drought stress while 58 NAC genes of Solanum muricatum that responded to drought stress had obvious tissue expression specificity. The overall expression levels in the root were found to be high. The number of genes at extremely significant and significant expression levels was very large, with significant polarization. Seven NAC genes with significant responses were selected to analyze their expression trend in the different drought stress gradients. It was found that genes with the same expression trend also had the same or part of the same conserved domain. Seven SmNACs that may play an important role in drought stress were selected for NAC amino 
acid sequence alignment of Solanaceae crops. Four had strong similarity to other Solanaceae NAC amino acid sequences, and SmNAC has high homology with the Solanum pennellii. The NAC transcription factor family genes of Solanum muricatum showed strong structural conservation. Under drought stress, the expression of NAC transcription factor family genes of Solanum muricatum changed significantly, which actively responded to and participated in the regulation process of drought stress, thereby laying foundations for subsequent in-depth research of the specific functions of NAC transcription factor family genes of Solanum muricatum. 


\section{Transcriptome-wide and Expression Analysis of the NAC \\ 3 Gene family in Pepino (Solanum muricatum) during drought}

\section{4 stress}

5 Shipeng Yang ${ }^{1, \dagger}$, Haodong Zhu ${ }^{2, \dagger}{ }^{\dagger}$, Liping Huang ${ }^{1}$, Guangnan Zhang ${ }^{1}$, Lihui Wang ${ }^{1}$, Xiaoting Jiang ${ }^{3}$ and

6 Qiwen Zhong ${ }^{1, *}$

$7 \quad{ }^{1}$ Qinghai Key Laboratory of Vegetable Genetics and Physiology, Agriculture and Forestry Sciences Institute of Qinghai

8 University, Qinghai University, Xining, P.R. China

92 Qinghai University, Xining, P.R. China

$10 \quad{ }^{3}$ Qinghai Higher Vocational \& Technical Institute, Ledu, P.R. China

11 * Correspondence: jerusalemyys@aliyun.com(Q.-W.Z.); Tel.: +86-13997135755

$12 \uparrow$ These authors contributed equally to this work.

13 Received: date; Accepted: date; Published: date

Abstract: Solanum muricatum (Pepino) is an increasingly popular solanaceous crop and is tolerant of drought conditions. In this study, 71 NAC transcription factor family genes of Solanum muricatum were selected to provide a theoretical basis for subsequent in-depth study of their regulatory roles in the response to biological and abiotic stresses, and were subjected to whole-genome analysis. The NAC sequences obtained by transcriptome sequencing were subjected to bioinformatics prediction and analysis. Three concentration gradient drought stresses were applied to the plants, and the target gene sequences were analyzed by qPCR to determine their expression under drought stress. The results showed that the Solanum muricatum NAC family of contains 71 genes, 47 of which have conserved domains. The protein sequence length, molecular weight, hydrophilicity, aliphatic index, and isoelectric point of these transcription factors were predicted and analyzed. Phylogenetic analysis showed that the Solanum muricatum NAC gene family is divided into seven subfamilies. Some NAC genes of Solanum muricatum are closely related to the NAC genes of Solanaceae crops such as tomato, pepper, and potato. The seedlings of Solanum muricatum were grown under different gradients of drought stress conditions and qPCR was used to analyze the NAC expression in roots, stems, leaves, and flowers. The results showed that 13 genes did not respond to drought stress while 58 NAC genes of Solanum muricatum that responded to drought stress had obvious tissue expression specificity. The overall expression levels in the root were found to be high. The number of genes at extremely significant and significant expression levels was very large, with significant polarization. Seven NAC genes with significant responses were selected to analyze their expression trend in the different drought stress gradients. It was 
of Solanaceae crops. Four had strong similarity to other Solanaceae NAC amino acid sequences, and SmNAC has high homology with the_Solanum pennellii .The NAC transcription factor family genes of Solanum muricatum showed strong structural conservation. Under drought stress, the expression of NAC transcription factor family genes of Solanum muricatum changed significantly, which actively responded to and participated in the regulation process of drought stress, thereby laying foundations for subsequent in-depth research of the specific functions of NAC transcription factor family genes of Solanum muricatum.

Keywords: NAC, drought stress, Solanum muricatum, gene expression, gene regulation

\section{Introduction}

Solanum muricatum is a perennial plant of the nightshade family, Solanaceae, native to the Andes in South America. Compared with most fruits, Solanum muricatum is rich in potassium, vitamin C, and selenium (Kola 2010). The flesh is delicious and it has fragrance and succulence. Antioxidants in Solanum muricatum have been found to have medicinal value, such as lowering blood pressure, diuresis, preventing alcoholic fatty liver, and anti-cancer properties (Cheng-chin et al. 2011; Kola et al. 2015; Shathish \& Guruvayoorappan 2014; Shathish et al. 2015). Solanum muricatum is now cultivated around the world and is being gradually recognized as an important medicinal plant. At present, China has planted a large number of Solanum muricatum in Qinghai, Gansu, Yunnan, and other places, growing in high-altitude areas around 1000-3000 meters above sea level. Abiotic stress factors such as high temperature, drought (Langeroodi et al. 2020; Zamarud et al. 2020), and biotic factors such as diseases and insect pests (C et al. 2020; Tadashi \& Ken 2019) have caused serious damage to the plant growth, development, and production. These stress factors restrict the development and output in the industries dealing with Solanum muricatum.

Solanum muricatum is suitable for planting in relatively arid and barren areas; Jiuquan, Wuwei, and other places in the main planting areas of west Gansu are all Gobi desert areas. The main cultivation area of Yunnan contains the Stone Forest, part of the karst landform, characterized by rugged geology and poor soil nutrients (Tadashi \& Ken 2019). The frequent occurrence of extreme climates around the world has exacerbated the adverse effects of abiotic stress on agricultural production, especially drought stress. The primary stress effect caused by drought is high osmotic stress, usually referred to as osmotic stress. The secondary effects of drought stress are more complex and, although these may eventually lead to plant metabolic disorders, the most important consequence of drought stress is the accumulation of the plant hormone ABA as a result of osmotic stress, that, in turn, affects the adaptive response of the plant. Studies have shown that regulation of the NAC transcription factor family under drought stress enhances the osmotic tolerance ability of the plant "store" and senescent leaves that have lost their osmotic tolerance act as "sources". In this way, water in the plant is preferentially transported 
67 to the "store" to promote plant survival and the continuation of the species (Chen et al. 2018; Jinxiang et al. 2016). Besides this, NAC family genes have been shown to regulate a wide range of developmental processes, including fiber development (Shim et al. 2018), cell wall synthesis (Huang et al. 2015), cell expansion (Pei et al. 2013; Yunjun et al. 2014), leaf senescence, and fruit ripening (Xuemin et al. 2018). Further, plant NAC transcription factors also play an important regulatory role in response to adverse stress conditions including drought (Yuan et al. 2019). It has been reported that alterations in the expression of NAC transcription factors can increase the plant's tolerance to abiotic stress (Yong et al. 2019).

NAC transcription factors, as a plant-specific superfamily that is involved in various developmental processes, including stress response and tolerance (Kazuo et al. 2012), have been intensively investigated in recent years. The number of NAC transcription factors varies between 30-200 among different crops. There are 33 transcription factors in Physcomitrella patens (Rakhi \& Swarnendu 2019), 63 in coffee (Coffea canephora) (Dong et al. 2019; Mao et al. 2016), 87 in sesame (Sesamum indicum) (Zhang et al. 2018), 104 in pepper (Capsicum annuum L.) (Weiping et al. 2018), and 114 in Betula pendula (Shen et al. 2019; Weiping et al. 2018). There has been a great deal of research on the NAC in Solanaceae crops. In the tomato (Solanum lycopersicum), the NAC family member JUB1 interacts directly with the promoters of SIDREB1, SIDREB2, and SIDELLA, increasing the drought tolerance of tomato. JUB1 acts as a transcriptional regulator of drought tolerance and controls the regulatory network of abiotic stress-related genes, which is fairly conserved among Arabidopsis thaliana and tomato (Alshareef et al. 2019). Similarly, the NAC transcription factors SINAC35 and SINAC11 are involved in the response to drought stress and salt stress in tomato. Overexpression of SINAC35 has shown that it can affect the growth and development of the roots, as well as promoting the expression of members of the ADP ribosylation factor (ARF) family, indicating that NAC have a role in mediating auxin conduction and regulation (Wang et al. 2016; Wang et al. 2017). In the potato (Solanum tuberosum L.), StNAC002, StNAC025, StNAC087, and StNAC091 enhance resistance while several NAC can enhance the disease resistance of potato to anti-phytophthora (Singh et al. 2013). There are 176 NAC (NtNAC) transcription factors in tobacco (Nicotiana tabacum L.), the earliest crop to be used for conducting functional studies of the NAC family, although there have been few conclusive reports (Tweneboah \& Oh 2017). NtNACR1 increases lateral root development and nicotine content during tobacco topping, while NtNAC2 increases the salt resistance of plants (Han et al. 2014). Compared to tomato and other Solanaceae crops, NAC proteins in pepper have somewhat longer sequences. CaNAC2 is mainly expressed in seeds and roots. The silencing of CaNAC2 verifies that this gene has a positive regulatory effect on the plants during cold and salt stress (Wei-Li et al. 2015).

Because of their prominent role in plant abiotic stress, growth, and development, the NAC gene family is an important focus of research. Solanum muricatum is similar to the other solanaceous crops originating from the

100 Andes. However, although other solanaceous have been intensively studied, the research on Solanum muricatum is only about 30 years old. This research shows that Solanum muricatum has high stress-resistance and excellent 
102 planting prospects in saline-alkaline and arid areas (Duman \& Sivaci 2015; Jinxiang et al. 2016; Prohens et al.

103 2002). The mechanism of Solanum muricatum's resistance against drought has not been investigated. The use

104 of biotechnology to regulate a plant's NAC transcription factors could enhance its resistance to adverse 105 environments and would be of great significance for production. The exploration and analysis of the NAC gene 106 family can provide technical support for Solanum muricatum breeding and cultivation. In this study, the NAC 107 transcription factor family genes of Solanum muricatum were identified and analyzed, investigating their 108 evolutionary classification, protein physicochemical properties, conserved domains, tissue-specific expression, 109 and expression of conserved domain genes under different degrees of drought stress. The study aims to provide 110 a theoretical basis for further exploration of the biological function and regulatory mechanisms of the NAC genes 111 of Solanum muricatum.

112

\section{2. Materials and Methods}

\subsection{Identification and biological analysis of SmNAC gene family}

115 The Solanum muricatum sequencing data were obtained from Illumina HiSeq 2000 sequencer (Accession: 116 SRX1181733, SRX1177957). Using BMKCloud (https:/ 117 /international.biocloud.net/zh/project/detail/119213). The Trinity assembly of transcripts was carried out by 118 the Eukaryotic Transcriptome Analysis Platform. A total of 68891 unigenes were obtained, and the N50 was 119 1415. The integrity of the assembly was good. We investigated these unigenes further using the NCBI nr 120 (https://www.ncbi.nlm.nih.gov/refseq/about/nonredundantproteins/), Gene Ontology (GO; 121 http://geneontology.org/), Kyoto Encyclopedia of Genes and Genomes (KEGG; https://www.genome.jp/kegg/), 122 and other databases. All NAC genes in Solanum muricatum were searched. The amino acid sequences 123 corresponding to all the transcripts of Solanum muricatum were obtained by ORF prediction 124 (http://bioinformatics.ysu.edu/tools/Orf Predictor.html). The amino acid data were uploaded to Pfam 125 (http://pfam.sanger.ac.uk/) for gene function annotation, and finally, a total of 71 NAC nucleotide sequences 126 with NAM and NAC functional domains of Solanum muricatum were retrieved for further analysis. The NAC 127 protein sequences of Arabidopsis, rice, tomato, potato, and pepper were compared with the protein sequences of 128 Solanum muricatum. All Solanum muricatum transcript sequence numbers with high similarity to NAC protein 129 sequences (e-value < -50) were identified (F. et al. 1997). The online software ExPASy Translate tool 130 (https://web.expasy.org/translate/) was used to translate the SmNAC nucleotide sequences into amino acid 131 sequences, and the Compute pI / MW tool was used on the ExPasy (http://au.expasy.org/tool.html) for analysis 132 to obtain the various physical and chemical properties of the SmNAC proteins, including length, molecular 133 weight, cDNA length, aliphatic index, and isoelectric point. The SmNAC proteins were analyzed for conserved 134 domains through the MEME website (http://meme-suite.org), and TBtools was used to draw domain site maps 135 (Chen et al. 2020). 


\section{$137 \quad 2.2$ Construction of phylogenetic tree}

138 NAC family protein sequences of Arabidopsis were downloaded from the Plant Transcription Factor 139 Database (http://planttfdb.cbi.pku.edu.cn/), and 24 tomato (Solanum lycopersicum) NAC genes, 27 potato 140 (Solanum tuberosum L.) NAC genes and 25 pepper (Capsicum annuum L.) NAC genes were downloaded from 141 the NCBI database (https://www.ncbi.nlm.nih.gov/) for inclusion in the construction of the phylogenetic tree

142 along with the 71 Solanum muricatum NAC genes. The gene sequence fasta files are shown in Supplementary 143 File 1. MUSCLE (http://www.drive5.com/muscle/) was used to perform multiple alignments of theSolanum 144 muricatum, Arabidopsis, pepper, potato, and tomato NAC amino acid sequences. The MEGA 7.0 software and 145 maximum likelihood method (ML, bootstrap = 1500) were used to construct an unrooted phylogenetic tree 146 (Sudhir et al. 2018) and the online software iTOL (https://itol.embl.de/) was used to annotate the tree.

\section{$148 \quad 2.3$ Tissue-specific expression of SmNAC}

\section{$149 \quad$ 2.3.1 Plant materials and drought stress}

150 Detoxified Solanum muricatum plants planted during the same period were placed in clean water and cultivated for two days in a $24^{\circ} \mathrm{Cconstant}$ temperature incubator with $16 \mathrm{~h}$ of light and $70 \%$ humidity. After this

152 time a sample of leaves from the plants was cut and frozen at $-80^{\circ} \mathrm{C}$ as a control group for qPCR assay. Then, 153 the plants were transferred into a 15\% PEG2000 solution and cultured for two days under the same conditions. 154 The roots, stems, leaves, and flowers of the plants were cut and frozen at $-80^{\circ} \mathrm{C}$, which was used as an 155 experimental group for subsequent qPCR assay. Three independent experiments were conducted.

\section{$157 \quad$ 2.3.2 Fluorescence quantitative analysis of gene expression}

158 The leaves from plants grown without drought stress were used as controls, while the roots, stems, leaves, 159 and flowers of plants grown in 15\% PEG2000 solution stress for two days were subjected to qPCR assay for the $16071 \mathrm{SmNAC}$ genes. Primer Premier 6.0 (http://www.premierbiosoft.com/primerdesign/) was used to design the 161 qPCR primers for SmNAC genes. TIANGEN RNAprep Pure polysaccharide polyphenol plant total RNA 162 extraction kit (DP441; TIANGEN, Beijing, China) was used to extract RNA, and TIANGEN miRNA cDNA 163 first-strand synthesis kit (kr211) was used to synthesize the first strand of cDNA. The cDNA of each sample was 164 diluted to $100 \mathrm{ng} / \mu \mathrm{L}$ and used as a template. The APT121 (adenine phosphoribosyl transferase) gene of Solanum 165 muricatum was used as the internal reference gene. qPCR amplification was performed using the TIANGEN 166 SuperReal PreMix Plus (SYBR Green) (FP205) kit. The total volume of the reaction was $20 \mu \mathrm{L}$, containing 10 $167 \mu \mathrm{L}$ SYBR green supermix, $7.8 \mu \mathrm{L} \mathrm{ddH_{2 }} \mathrm{O}, 0.6 \mu \mathrm{L}$ for each of the forward and reverse primers and $1.0 \mu \mathrm{L}$ cDNA. 
168 The reaction conditions were $95^{\circ} \mathrm{C}$ pre-denaturation for $15 \mathrm{~min} ; 95^{\circ} \mathrm{C}$ denaturation for $10 \mathrm{~s}$, annealing for $20 \mathrm{~s}$, 169 and with a total of 45 cycles. The $2-\triangle \Delta \mathrm{CT}$ method was used to calculate the relative expression of the target 170 genes. Omishare Tools heatmap (https://www.omicshare.com/tools/Home/Soft/heatmap) was used to draw the 171 heatmap. A single SmNAC was treated as an object, the expression levels in each tissue were normalized, and 172 each row and column of the heatmap was clustered.

173

$174 \quad 2.4$ Differential expression of SmNAC genes under different degrees of drought stress

$175 \quad$ 2.4.1 Plant materials and drought stress

176 Seven-week-old detoxified Solanum muricatum seedlings were placed in clean water and cultured in a $24^{\circ} \mathrm{C}$ 177 constant temperature incubator for two days with $16 \mathrm{~h}$ of light and $70 \%$ humidity, and then transferred to 178 PEG2000 solutions with concentrations of 5\%,10\%, and 15\% for culture under the same conditions. After $0 \mathrm{~h}$, $1794 \mathrm{~h}, 8 \mathrm{~h}, 12 \mathrm{~h}, 18 \mathrm{~h}, 24 \mathrm{~h}$, and $32 \mathrm{~h}$ of drought stress, the leaves were frozen at $-80^{\circ} \mathrm{C}$. The experiments were repeated 180 thrice.

\subsubsection{Fluorescence quantitative analysis of gene expression}

Based on the tissue-specific expression of the 71 SmNACs and the analysis of conserved domains, SmNAC3, SmNAC4, and SmNAC8 were selected. These genes had significant expression in the leaves and were comprised of five identical conserved domains (Motifs 13, 20, 6, 15, 3). SmNAC83 with five conserved domains (Motifs 1, 2, 5, 17, 4) was used as a reference, while the clusters SmNAC100 (with Motifs 1, 5, and 17), SmNAC91 (with Motifs 1 and 2) and SmNAC69 (with Motifs 2 and 5) containing sections of the identical domains were selected. The expression level of each gene at $0 \mathrm{~h}$ was set as a control, and the Solanum muricatum APT121 gene was set as an internal reference gene. The qPCR assay was performed for the above seven SmNAC genes. The qPCR experimental method was the same as that in section 2.3.2. The seven SmNAC gene-specific primer sequences and their annealing temperatures are shown in Table 1. GraphPad Prism8 was used to draw a histogram representing the levels of gene expression.

194 Table 1. The information of upstream and downstream primers used for the screening of seven SmNAC genes and the annealing temperature set during qPCR assay. 
198

199

200

201

202

203

204

205

206

207

208

209

210

211

212

213

214

215

216

217

218

219

220

221

222

223

224

225

226

227

228

229

The seven SmNACs screened in response to drought stress in section 2.4 were aligned with the other solanaceous sequences using BLAST-P (https://blast.ncbi.nlm.nih.gov). It was found that the four NAC amino acid sequences, SmNAC83, SmNAC91, SmNAC100, and SmNAC69, showed good alignment with other Solanaceae NAC family members, and had a high degree of similarity to the members of the NAC family of peppers, potatoes, tomatoes, and tobacco. The NAC gene sequences of these Solanaceae crops were downloaded and grouped to the same group with the corresponding SmNAC genes. The online software ExPASy Translate tool (https://web.expasy.org/translate) was used to translate the downloaded sequences. Multiple sequence alignment by CLUSTALW (https://www.genome.jp/tools-bin/clustalw) was performed on the four groups of NAC amino acid sequences, and the alignment results were presented using Jalview software (https://www.jalview.org/).

\section{Results}

\subsection{Identification of SmNAC gene family members and protein structural analysis}

In this experiment, a total of 71 Solanum muricatum NAC nucleotide sequences with NAM and NAC functional domains were retrieved for analysis. The analysis of the physical and chemical properties of the SmNAC genes using ExPasy showed that the protein sequences of the different NAC transcription factors were quite different, the amino acid lengths ranged from $64 \sim 1201$ aa, with an average value of 312.04 aa, while the protein molecular weight was $7.73 \sim 139.15 \mathrm{kDa}$, with an average value of $36.18 \mathrm{kDa}$ (Table 2). Among them, SmNAC63 had the shortest amino acid length, with only 64 aa, and SmNAC70 had the longest amino acid length reaching 1201 aa. SmNAC41 had the highest isoelectric point at 10.83, and SmNAC68 had the lowest isoelectric point at 4.67. Among them, there were 11 NAC proteins with relatively acidic isoelectric points of less than 7. The isoelectric points of the remaining 60 NAC proteins were all greater than 7 and, thus, relatively alkaline. The hydrophilicity of SmNAC16 was the lowest at $\sim-1.311$, and that of SmNAC9 was the highest at $\sim 0.732$. Out of the 71 NAC proteins, 40 proteins had a hydrophilicity value of less than 0 , which were hydrophilic, while 31 proteins had a hydrophilicity value of greater than 0 , making them hydrophobic.

Table 2. Structural, physical, and chemical properties of the 71 Solanum muricatum NAC gene family proteins, including the length of cDNA, amino acid length, protein molecular mass, isoelectric point, hydrophilicity, and aliphatic amino acid index of each SmNAC.

\subsection{Conserved domain analysis of SmNAC gene family}

Peer) reviewing PDF | (2020:06:49879:3:0:NEW 23 Dec 2020) 
Conserved domain analysis was performed on the entire sequences of the 71 Solanum muricatum NAC 231 proteins. Forty-four SmNAC protein sequences were identified as having conserved domains (Motifs) (Figure 232 1). These conserved domains comprised 20 Motif categories (Figure 2). It can be seen from Figure 2 that Motifs

233 4, 12, and 8 had the highest frequency and appeared 10 times during the search. We observed that SmNAC3, 234 SmNAC4, and SmNAC8 had only five identical motifs. The relative positions of these motifs were fixed and 235 the distance was relatively close. This indicates conservation and suggests that the biological functions of 236 SmNAC3, SmNAC4, and SmNAC8 are likely to be similar. Motifs 10, 7, 12, and Motif 8 appeared together in 237 five SmNAC protein sequences. The relative position of the above motif was constant and close to the C238 terminus. Motifs 1, 2, 5, and Motif 17 were found together in four SmNAC protein sequences simultaneously, 239 two of which had only four motifs. The relative positions of these four motifs were constant and located near the 240 N-terminus.

242 Figure 1. The position of the 44 conserved domains of SmNAC protein on the amino acid chain. The rectangles 243 of different colors represent different motifs and their positions on each of the NAC sequences. The 244 correspondence between the rectangles of each color and motif is shown in the upper right corner of the figure. 245 The horizontal axis at the bottom of the figure is the reference axis of the length of the amino acid chain.

Figure 2. The 20 conserved motifs (Motifs 1 to 20) identified in Solanum muricatum NAC proteins. The length, degree of conservation, and the sharing or substitution of every amino acid in each of the motifs are displayed. Each motif had a corresponding coordinate axis, the abscissa represents the corresponding position of each amino acid on the motif, and the ordinate represents sections.

3.3 Phylogenetic analysis of SmNAC gene family

253

To better understand the phylogenetic relationships within the SmNAC gene family, the NAC protein sequences of Solanum muricatum (71 NAC protein sequences), Arabidopsis (30 NAC protein sequences), tomato (24 NAC protein sequences), potato (27 NAC protein sequences), and pepper ( 25 protein sequences) were subjected to multiple alignments to construct an unrooted evolutionary tree (Figure 3). Solanum muricatum is the target species of this experiment, so only the Solanum muricatum were divided into categories. According to their branch on the tree, the 71 SmNAC transcription factors were roughly divided into seven categories: Classes I, II, III, IV, V, VI, and VII, of which Classes II and III had only a single NAC gene, while Class II had 15 Solanum muricatum NAC genes and Class III had 20 Solanum muricatum NAC genes. These two major categories of Solanum muricatum genes had no obvious homology with sequences from other 
262 species. Class I contained 11 Solanum muricatum and 1 tomato NAC sequence, with these sequences showing 263 relatively high similarity. There were 17 Solanum muricatum NAC and 1 pepper NAC distributed in Class IV. 264 Classes VI and VII branched from the pepper NACs, which formed the main branch, containing 2 and 1 Solanum muricatum NAC, respectively, indicating that Solanum muricatum had a closer evolutionary relationship with pepper than with Arabidopsis and other solanaceous crops. SmNAC022 and SmNAC064 were found on independent branches. Class V was the largest branch in the evolutionary tree with 41 NACs, of which only 3 belonged to Solanum muricatum. This branch included all species participating in the alignment; a total of 4 Solanaceae NACs, including SmNAC045, SINACMTF2, CaNAC091, and StNAC078. These further formed a sub-branch of Class $\mathrm{V}$, with obvious homology in evolution and functional similarity.

Figure 3. Unrooted phylogenetic tree showing the relationship between the NAC family members of Arabidopsis, pepper, tomato, potato, and Solanum muricatum. Each species is represented by a background color as shown in the upper left corner of the figure. Classes of different colors represented different subfamilies of Solanum muricatum NAC.

\subsection{Effect of drought stress on the seedlings of Solanum muricatum}

To further understand the effects of drought stress on Solanum muricatum seedlings, this experiment used three concentrations $(5 \%, 10 \%$, and $15 \%)$ of PEG to simulate different degrees of drought stress, and observed the changes in Solanum muricatum seedlings under different degrees of drought stress (Figure 4). The results showed that under drought stress, the seedling leaves wilted and showed chlorosis, which appeared earlier in seedlings grown in higher PEG concentrations i.e. conditions of greater drought stress. Under mild drought stress (5\% PEG solution), the old leaves of the seedlings sagged significantly after $18 \mathrm{~h}$ with no obvious change in the whole plant from $18 \mathrm{~h}$ to $32 \mathrm{~h}$. Most of the leaves remained upright after $32 \mathrm{~h}$ of stress induction. Under moderate drought stress (10\% PEG solution), the leaves sagged around $12 \mathrm{~h}$ of exposure; the old leaves on the lower half of the seedlings wilted and curled after $18 \mathrm{~h}$, with an increased degree of wilting with time and resulting in complete chlorosis at around $32 \mathrm{~h}$. The leaves in the lower half wilted completely, while the leaves in the upper half remained unaffected. Under severe drought stress (15\% PEG solution), the older leaves on the lower half of the seedlings wilted and sagged after $12 \mathrm{~h}$ while the top leaves began to wilt at around $18 \mathrm{~h}$ of exposure. The 
295 Figure 4. Changes in the plant shape of seven-week-old Solanum muricatum seedlings after drought stress. The 296 drought stress experiment was conducted in an incubator at $24^{\circ} \mathrm{C}$ with $70 \%$ relative air humidity, and $16 \mathrm{~h} / \mathrm{d}$ light.

297 The red gradient deepening process indicates the passage of drought stress time, and the green gradient deepening 298 process indicates the increase of PEG solution concentration. The photos show the morphological changes of 299 Solanum muricatum plants at various sampling time points ( 4 h, 8 h, 12 h, 18 h, 24 h, and 32 h) and in the 300 presence of PEG at three different concentrations (5\%, 10\%, and 15\%).

\section{$301 \quad 3.5$ The tissue-specific expression of SmNAC genes}

302 To analyze the function of Solanum muricatum NAC genes under drought stress, qPCR was used to analyze 303 the expression of the 71 NAC genes in four different tissues and organs, namely, roots, stems, leaves, and 304 flowers. The differences in the expression of individual SmNAC in each tissue were analyzed and each row was normalized. The results showed that there were significant differences in the expression levels of different NAC genes in different tissues and organs of Solanum muricatum (Figure 5). A total of 13 SmNAC genes were not expressed in the above four tissues, indicating that they did not respond to drought stress, but may respond to other abiotic stresses or biological stresses. According to the normalization and clustering results of each row, the SmNAC gene expression in the root was more characteristic than that in other tissues. The number of genes with the highest or lowest expression in each tissue in the root was more than that of flowers, leaves, and stems.

311 The polarization was very obvious, that was, the amplitude of gene up-regulation or down-regulation was higher

312 than that of the other tissues. The number of SmNAC with the highest expression levels was relatively high in

313 flowers, which was second only to the roots, and the genes having an average expression level were maximum

314 in all the tissues. Only the expression level of SmNAC52 in flowers was significantly lower than in other tissues.

315 The clustering results of each column showed that the expression status of SmNAC in leaves and stems was 316 almost similar, and the number of genes at the highest expression level or the lowest expression level was 317 approximately equal. It is worth mentioning that SmNAC3, SmNAC4, and SmNAC8 had five identical 318 conserved domains and their expression in leaves was very significant. It can be speculated that these three genes 319 played a significant role during drought stress in Solanum muricatum plants, and they may have similar 320 biological functions.

Figure 5. Heatmap showing the expression of Solanum muricatum NAC genes in four tissues (root, stem, leaf, and flower) under drought stress. A positive value represents a higher than the average level, 0 the average level, and a negative value represents below-average levels. 
Figure 4 shows the real-object diagram of three kinds of PEG concentration simulating drought stress. With

328

329

330

331

332

333

334

335

336

337

338

339

340

341

342

343

344

345

346

347

348

349

350

351

352

353

354

355

356

357

358

the increase of the PEG solution concentration, the rate of wilting and chlorosis of Solanum muricatum plants was accelerated. Based on the tissue-specific expression and conserved domain analysis of the 71 SmNACs, seven SmNACs were selected for qPCR analysis to further explore the changes in SmNAC gene expression over time under different concentrations of PEG stress. As a general observation, as the of drought increased, expression (both upregulated and downregulated) of the SmNAC genes increased, reaching extreme values more rapidly (Figure 6). A gene expression level of $\geq 2$ or $\leq 0.5$ was regarded as a significant change in the gene expression level. The expression trends of SmNAC3, SmNAC4, and SmNAC8 were similar, and the expression curve showed a single peak curve. It is possible that these three proteins have similar biological functions. Among them, the expression levels of SmNAC3 was the highest among all the tested genes, reaching 46 times under mild drought stress and 135 times under severe drought stress. During the experimental sampling period, SmNAC8 had consistently higher expression levels under severe drought stress and the relative change was smaller than that for the other genes. The expression trends of SmNAC83, SmNAC100, and SmNAC91 were similar, and the expression curve was roughly bimodal, all reaching the first peak at $4 \mathrm{~h}$, then showing a downward trend and decreasing to the minimum at $18 \mathrm{~h}$ or $24 \mathrm{~h}$, with gradual increase thereafter. Among them, SmNAC100 responded to drought stress most significantly. The expression level under mild drought stress reached 45 times at $4 \mathrm{~h}$, and the expression level under severe drought stress reached 122 times. The relative expression levels of SmNAC83 and SmNAC91 between $18 \mathrm{~h}$ and $24 \mathrm{~h}$ were around 1, which can be regarded as the normal expression level, while SmNAC69 was a down-regulated gene. With the increase in the degree of drought stress, the minimum value was reached more rapidly, at $8 \mathrm{~h}$ under severe drought stress, and at $18 \mathrm{~h}$ under mild drought stress, with downregulation of the gene at almost $1 / 10$ of normal levels. The expression of SmNAC69 was significantly suppressed.

Figure 6. Changes in the expression of the selected seven Solanum muricatum NAC genes over time under different degrees of drought stress. ThreePEG concentrations (5\%,10\%, and 15\%) were used to stress the Solanum muricatum plants. Leaves were collected after 0 h, 4 h, 8 h, 12 h, 18 h, 24 h, and 32 h, and the expressions of Solanum muricatum NAC genes were detected by qPCR. The $2-\Delta \Delta \mathrm{CT}$ method was used to calculate the relative expression of the target genes. The abscissa represents each sampling time point of the experiment $(4 \mathrm{~h}, 8 \mathrm{~h}, 12 \mathrm{~h}, 18 \mathrm{~h}, 24 \mathrm{~h}$, and $32 \mathrm{~h}$ ). The ordinate indicates the relative expression levels. White, gray, and black represent $5 \%, 10 \%$, and $15 \%$ PEG stress experimental groups, respectively.

\subsection{NAC amino acid sequence alignment of various species}

Peer) reviewing PDF | (2020:06:49879:3:0:NEW 23 Dec 2020) 
The amino acid BLAST alignment results of the seven SmNACs (Fig. 6) that actively responded to drought stress were analyzed, and it was found that four NACs i.e. SmNAC83, SmNAC100, SmNAC91, and SmNAC69 showed relatively good alignment with the Solanaceae crops. The alignment results all included the same five species (Solanum pennellii, Solanum tuberosum, Capsicum annuum, Nicotiana tabacum, and Nicotiana tomentosiformis)(Figure7). Overall, these SmNAC showed strong homology with tomato, potato, pepper, and tobacco sequences.Tobacco crops showed strong specificity in the process of comparison. The amino acid comparison results showed that SmNAC69 was highly similar to tomato, potato, and pepper. The tobacco sequence, at 140 aa, had an additional two residues, resulting in a subsequent conservative conserved sequence shift; SmNAC83 had the highest similarity with tomato, and, among the fragments, the similarity of the sequences from the five plants in the range of 1-105 aa was high while different numbers of glutamine residues were seen in the different plant sequences beyond residue 105. SmNAC91 had the highest similarity with tomato and potato, and tobacco and pepper showed strong high specificity in this comparison. It is worth mentioning that the specificity of capsicum was the most prominent; the alignment of NAC100 was the best among the four groups of genes .Apart from the substitution of isolated amino acids residues, the sequences showed a high degree of conservation.

Figure 7. Multiple sequence alignments of four groups of NAC genes. The Zappo Colours color scheme (http://www.jalview.org/version118/documentation.html\#zappo) was used to present the amino acid sequence. Note: (Conservation: Conservation of total alignment of less than 25\% gaps. Quality: Alignment Quality based on Blosum62 scores. Consensus: PID (http://www.jalview.org/version118/documentation.html\#pid). Occupancy: Number of aligned positions.)

\section{Discussion}

\subsection{Bioinformatics analysis of Solanum muricatum NAC gene family}

NAC transcription factors are generally less than 400 amino acids long with highly conserved N-termini.

The protein sequence contains the approximately 150-residue NAC domain which is composed of five substructures: A, B, C, D, and E (Hisako et al. 2003). The lengths and sequences of the C-terminal regions are highly diverse (Xin-Jian et al. 2005). In this study, it was found that the N-terminus of SmNAC2, SmNAC44, SmNAC83, and SmNAC46 contained Motifs 2, 1, 5, and 17, while the C-terminus of SmNAC83 had Motif 4, and the SmNAC had Motifs 1, 5, and 7, which is consistent with the characteristic NAC features of a highly conserved N-terminus and a diversified C-terminus (Yujie et al. 2008). However, there were certain differences in the N-terminal conserved domains. For example, SmNAC3, SmNAC4, and SmNAC8 had five identical 
393 Arabidopsis, with a highly conserved NAC domain at the N-terminus (Swati et al. 2012). The SmATAF2

394 identified in this study had the same Motif 2, 1, and 5 at the N-terminus as SmNAC2. It also had the same Motif

39520 as SmNAC3 and other sets of genes. It has also been reported that the N-terminal domains of the Solanaceae

396 pepper and potato NAC families are not completely consistent (Schafleitner et al. 2013; Weiping et al. 2018).

397 These findings indicate that the N-termini of NAC transcription factors are not completely conserved.

398 The phylogenetic tree showed that the 71 members of the NAC family of Solanum muricatum can be divided 399 into seven subfamilies which are closely related to the NACs of pepper in the Solanaceae family. From previous 400 studies on the evolution of the NAC family, it has been found that there is an evolutionary relationship between 401 Arabidopsis and the solanaceous crops such as peppers, potatoes, and tomatoes (Tweneboah \& Oh 2017). Class

$402 \mathrm{~V}$ in the evolutionary tree constructed in this study also supports this phenomenon. In the evolutionary analysis 403 of the pepper NAC family, it was found that there was a branch containing only pepper NAC (Schafleitner et al. 404 2013), and a similar situation was observed while constructing the Class II and Class III evolutionary tree. This 405 Solanum muricatum NAC does not seem to have a close relationship with NACs other species, which suggests 406 that they may play a unique role in the growth and development of Solanum muricatum. NACMTFs are unique 407 transmembrane proteins in plants. After receivingstress signals, they are transferred to the plasma membrane and 408 truncated at the C-terminus for translocation either into the cytoplasm or nucleus, thereby completing the 409 transmembrane signal transmission. This pathway has been reported in Arabidopsis and other crops (Kim et al. 410 2007). In potato and tomato, which also fall in the Solanaceae family, several NACMTFs have been identified 411 (Bhattacharjee et al. 2017; Schafleitner et al. 2013). SINACMTFs are tomato-specific membrane-binding 412 factors, and in this study, it was found that SmNAC45 and SmNAC40 were closely related to SINACMTF2 and 413 SINACMTF3, suggesting that they may have similar biological functions. It is worth mentioning that 414 SINACMTFs will significantly affect the activity of the NAC promoter. The same SINACMTF shows different 415 regulatory modes for different promoters (Bhattacharjee et al. 2017), but whether SmNAC40 and SmNAC45 416 follow the same regulatory pathway needs further research.

\subsection{Expression of Solanum muricatum NAC transcription factors under drought stress}

According to the research by Han et al., the expression levels of the SINAC3 gene in the flowers and roots of tomato were relatively high, indicating that the growth of flowers and roots was under the influence of this transcription factor (Qinqin et al. 2012). Also, the expression of SmNAC9, SmNAC55, SmNAC25, SmNAC59, and SINAC3 was similar, which indicates that they may be closely related to the growth and development of flowers and roots. It is worth mentioning that SINAC3 has a diversity of functions. Salt stress, drought stress, and ABA treatment can inhibit the expression of SINAC3. The analysis results showed that SINAC3 may interact with environmental and endogenous stimuli and act as a transcriptional activator in plants through the ABA 
427

428

429

430

431

432

433

434

435

436

437

438

439

440

441

442

443

444

445

446

447

448

449

450

451

452

453

454

455

456

457

458

459

460

functional prediction and verification of SINAC3 can provide research directions for future research on the functions of the SmNAC genes.

In this study, while investigating the tissue-specific expression of SmNAC under drought stress, we found that, compared with other tissues, the highest expression of the upregulated SmNACs was in the roots, followed by the flowers, while most of the genes showed average expression levels. In general, the expression levels were significantly upregulated, consistent with the hypothesis that flowers are the main source of water loss during drought stress compared to the stems and leaves (Ibrahim et al. 2020). There were many genes showing the highest and lowest expression levels in the roots, where significant polarization was observed, in other words, the amplitude of the gene upregulation or downregulation was greater than in other tissues, with SmNAC23, SmNAC40, and others being typical representatives. In potato, the expression of StNAC103 in the roots was significantly higher than that of other genes (Schafleitner et al. 2013); StNAC103 has been shown to be a repressor of suberin polyester and suberin-associated wax deposition (Soler et al. 2020). Whether SmNAC23 and other genes also have related functions needs to be further confirmed. In research on the potato NAC family, it was found that StNAC33, StNAC55, and StNAC97, which had seven identical motifs and are closely related, had similar expression trends in tissue-specific expression (Schafleitner et al. 2013). Similar findings were found in the tissue-specific expression of SmNACs 3, 4, and 8 with five identical motifs and all belonging to Class III, which showed greater expression in the leaves compared to the other tissues. It is speculated that these three SmNACs with the same motif may execute the same biological function in the leaves.

\subsection{Regulation of NAC transcription factors under abiotic stress}

In recent years, there has been much research on the NAC gene family. Many species have been used for the identification and functional prediction of the NAC gene family. The latest research on Moso bamboo (Phyllostachys edulis) identified 94 NAC genes, of which 15 PeNACs played a significant role in secondary cell wall biosynthesis and lignification of the bamboo (Xuemeng et al. 2019). MdNAC52 and MdNAC42 in apple (Malus domestica) can regulate the synthesis and accumulation of anthocyanins (Shuangyi et al. 2020; Sun et al. 2019b). Likewise, 183 NAC genes were identified in the white pear (Pyrus bretschneideri), which were divided into 33 subgroups, out of which the $\mathrm{C} 2 \mathrm{f}, \mathrm{C} 72 \mathrm{~b}$, and $\mathrm{C} 100 \mathrm{a}$ subgroups actively responded to drought and lowtemperature stress (Xin et al. 2019). With increased research on the biological functions of NAC transcription factors, it has been shown that NAC transcription factors can regulate a wide range of plant life processes including plant growth and development, the growth and senescence of the cell wall and root through plant hormone signaling pathways (Wei et al. 2018; Yongil et al. 2019), and improving plant resistance by responding to a variety of abiotic and biological stress factors (Du et al. 2020; Jia et al. 2019). In terms of abiotic regulation, the research on the pathways and mechanisms of NAC family genes for abiotic stress has been intensively studied. For example, the NAC11 gene of Ammopiptanthus mongolicus encodes a transcription factor that can

Peer) reviewing PDF | (2020:06:49879:3:0:NEW 23 Dec 2020) 
461 respond to drought, low temperature, high salt, and other stresses. The translated protein is localized in the 462 nucleus and plays an important role in drought, cold, and salt tolerance (Xinyue et al. 2019). In the NAC family 463 of corn (Zea mays L.), overexpression of ZmNAC 071 in Arabidopsis enhanced the sensitivity of transgenic 464 plants to ABA and osmotic stress (Lin et al. 2019). Meanwhile, studies on splicing variants of the NAC family 465 have been carried out in corn (Zhang et al. 2019). In the Solanaceae family to which the Solanum muricatum 466 belongs, CaNAC064 of the pepper NAC family interacts with low-temperature-induced plant cell single-chain 467 protease proteins to positively regulate cold tolerance in plants. Analysis of the protein structure and expression 468 pattern of NtNAC2 in tobacco has been completed (Han et al. 2014). The research of these closely related species 469 has provided a good foundation for the follow-up research of the Solanum muricatum NAC family.

470 Although previous studies reported the association of the NAC gene family with abiotic stress, our experiments 471 are a more detailed exploration. Here we investigated the expression trends and functional prediction of the NAC 472 genes of Solanum muricatum under drought stress. The results showed that the expression levels of SmNAC83 473 and SmNAC91 initially increased, then fell back to normal levels before increasing again. This is very similar 474 to the reported expression trend of SINAC8 in the tomato NAC family under PEG drought stress where increases 475 were associated with higher degrees of stress (Kou et al. 2017). The expression level of SmNAC69 first showed 476 a decreasing trend and then gradually recovered to the normal expression level, which is similar to the reported 477 expression trend of SINAC9 under PEG drought stress (Kou et al. 2017). Subsequent studies in the tomato NAC 478 family have confirmed that the SINAC9 gene is involved in carotene synthesis and fruit softening of tomato 479 (Kou et al. 2018). Whether SmNAC69 has the same biological function is still unknown and hence it is necessary 480 to verify the specific function and mechanism of this gene in future research using either transgenic or gene 481 silencing technology. In the genome-wide analysis of the pepper NAC transcription factor gene family, CaNAC 482 showed three expression trends under drought stress. There are upregulated genes such as CaNAC23, CaNAC53, 483 CaNAC37 that exhibit a single peak curve, upregulated genes such as CaNAC72 and CaNAC79 that exhibit a 484 double peak curve, as well as downregulated genes such as CaNAC56, CaNAC33, CaNAC35 (Weiping et al. 485 2018). The seven SmNACs selected in this study also had three expression trends i.e. SmNAC3, SmNAC4, and 486 SmNAC8 are single-peak upregulated genes, SmNAC83, SmNAC100, and SmNAC91 are double-peak 487 upregulated genes, and SmNAC69 is a downregulated gene. This similar expression trend indicates that they 488 may also have similarities in their functions. In addition to the results of evolutionary tree analysis, this also 489 provides evidence for the strong homology between SmNAC and CaNAC. It is worth mentioning that both 490 CaNAC35 and SmNAC69 are downregulated genes and the expression trend is almost the same. CaNAC35 has 491 been shown to be a positive regulator of tolerance in response to abiotic stress in pepper and functions through 492 multiple signaling pathways (Huafeng et al. 2020). This can help in providing research directions for functional 493 verification of SmNAC69 in the future. 


\subsection{NAC amino acid sequence alignment of related species}

496 In general, SmNAC has high homology with the Solanum pennellii, Solanum tuberosum, Capsicum annuum,

497 Nicotiana tabacum, and Nicotiana tomentosiformis NAC families with the highest degree of similarity between

498 SmNAC and SpNAC. The results showed that orthologous NACs in each species were conserved with small

499 differences with the differences caused by subtle alterations such as the addition or replacement of individual

500 amino acids. SmNAC100, SpNAC100, StNAC100, CaNAC100, NtNAC100, and NtoNAC100 have high

501 degrees of similarity, which may be derived from the same ancestral NAC gene. As SmNAC100 is the most

502 similar gene to Nicotiana NAC among the four SmNAC genes, and tobacco is also a popular crop for functional

503 verification of NAC genes (Sun et al. 2019a; Wang et al. 2020), we will use SmNAC100 as the preferred gene

504 for functional verification in tobacco crops in the next step. The functional verification of NAC family genes in

505 Solanaceae, which are more closely related to SmNAC such as Capsicum annuum NAC1 and Solanum

506 lycopersicum NAC2, also provides theoretical guidance for the further study of SmNAC functions (Borgohain

507 et al. 2019; Xi-Man et al. 2020). In this study, the role of NAC genes in ginseng fruit under drought stress was

508 preliminarily discussed and compared with Solanaceae crops. However, the functional verification of these genes

509 needs to be further investigated in future research.

\section{5. Conclusions}

512 Using genome-wide identification of the Solanum muricatum NAC transcription factor family, we 513 identified a total of 71 family members belonging to seven subfamilies. These proteins had typical NAC 514 conserved domains and their gene expression was significantly influenced by drought stress. The expression

515 levels were relatively high in roots and flowers while expression in the leaves and stems was very similar. The 516 expression levels of certain genes in leaves showed three different trends: single peak upregulated expression, 517 double peak upregulated expression, and downregulated expression. The Solanaceae NAC amino acid sequence 518 alignment showed a high degree of homology, and Solanum muricatum NACs were strongly homologous with 519 NACs from pepper, tomato, potato, tobacco, and other Solanaceae crops.

520 Supplementary Materials: The following are available online.

Author Contributions: Conceptualization, Shipeng Yang and Haodong Zhu; Data curation, Haodong Zhu; Funding acquisition, Shipeng Yang and Qiwen Zhong; Investigation, Guangnan Zhang; Project administration, Shipeng Yang and Qiwen Zhong; Software, Haodong Zhu, Huangli Ping and Xiaoting Jiang; Supervision, Shipeng Yang and Lihui Wang; Validation, Shipeng Yang and Haodong Zhu; Writing - original draft, Haodong Zhu; Writing - review \& editing, Shipeng Yang and Qiwen Zhong.

Funding: This research was supported by the Association of Qinghai Science and Technology (2019QHSKXRCTJ06). The Key Laboratory Project of Qinghai Science \& Technology Department (2020-ZJ-Y02). Qinghai Agriculture and Forestry Science Innovation Fund(2020-NKY-02).

528 Conflicts of Interest: The authors declare no conflict of interest. 


\section{References}

531 Alshareef NO, Wang JY, Ali S, Al-Babili S, Tester M, and Schmöckel SM. 2019. Overexpression of the NAC transcription

532

533

534

535

536

537

538

539

540

541

542

543

544

545

546

547

548

549

550

551

552

553

554

555

556

557

558

559

560

561

562

563

564

565

566

567 factor JUNGBRUNNEN1 ( JUB1 ) increases salinity tolerance in tomato. Plant Physiology and Biochemistry 140.

Bhattacharjee P, Das R, Mandal A, and Kundu P. 2017. Functional characterization of tomato membrane-bound NAC transcription factors. Plant Molecular Biology 93.

Borgohain P, Saha B, Agrahari R, Chowardhara B, Sahoo S, Vyver C, and Panda SK. 2019. S1 NAC2 overexpression in Arabidopsis results in enhanced abiotic stress tolerance with alteration in glutathione metabolism. Protoplasma 256.

C A, P RM, M J, and P G. 2020. Long-Term Cocirculation of Two Strains of Pepino Mosaic Virus in Tomato Crops and Its Effect on Population Genetic Variability. Phytopathology 110.

Chen C, Chen H, Zhang Y, Thomas HR, Frank MH, He Y, and Xia R. 2020. TBtools-an integrative toolkit developed for interactive analyses of big biological data. bioRxiv:289660.

Chen H-H, Qu L, Xu Z-H, Zhu J-K, and Xue H-W. 2018. EL1-like Casein Kinases Suppress ABA Signaling and Responses by Phosphorylating and Destabilizing the ABA Receptors PYR/PYLs in Arabidopsis. Molecular Plant 11.

Cheng-chin H, Yu-ru G, Zhi-hong W, and Mei-chin Y. 2011. Protective effects of an aqueous extract from pepino (Solanum muricatum Ait.) in diabetic mice. Journal of the science of food and agriculture 91.

Dong X, Jiang Y, Yang Y, Xiao Z, Bai X, Gao J, Tan S, Hur Y, Hao S, and He F. 2019. Identification and Expression Analysis of the NAC Gene Family in Coffea canephora. Agronomy 9.

Du Y, Zhao Q, Chen L, Yao X, Zhang W, Zhang B, and Xie F. 2020. Effect of drought stress on sugar metabolism in leaves and roots of soybean seedlings. Plant Physiology and Biochemistry 146.

Duman S, and Sivaci A. 2015. Investigation Of Drought Stress In Pepino (Solanum Muricatum Ait. Cv. Miski) Leaves. Pak J Bot 47:1621-1627.

F. AS, L. MT, A. SA, Jinghui Z, Zheng Z, Webb M, and J. LD. 1997. Gapped BLAST and PSI-BLAST: a new generation of protein database search programs. Altschul Stephen F;Madden Thomas L;Schäffer Alejandro A;Zhang Jinghui;Zhang Zheng;Miller Webb;Lipman David J 25.

Han, Qiao, Song, and Zhang. 2014. Structural analysis and tissue-specific expression patterns of a novel salt-inducible NAC transcription factor gene from Nicotiana tabacum cv. Xanthi. The Journal of Horticultural Science and Biotechnology 89.

Hisako O, Kouji S, Koji D, Toshifumi N, Yasuhiro O, Kazuo M, Kenichi M, Naoki O, Jun K, Piero C, Yoshihide H, Koji S, Keiichi K, Yoshinori T, Koji Y, and Shoshi K. 2003. Comprehensive analysis of NAC family genes in Oryza sativa and Arabidopsis thaliana. DNA research : an international journal for rapid publication of reports on genes and genomes 10 .

Huafeng Z, Fang M, Xinke W, Suya L, Haq SU, Xiaoming H, Yumeng Z, Dan L, Yuancheng M, Wei Z, Khan A, and Rugang C. 2020. Molecular and Functional Characterization of CaNAC035, an NAC Transcription Factor From Pepper ( Capsicum annuum L.). Frontiers in plant science 11.

Huang D, Wang S, Zhang B, Shang-Guan K, Shi Y, Zhang D, Liu X, Wu K, Xu Z, Fu X, and Zhou Y. 2015. A GibberellinMediated DELLA-NAC Signaling Cascade Regulates Cellulose Synthesis in Rice. The Plant Cell 27.

Peer) reviewing PDF | (2020:06:49879:3:0:NEW 23 Dec 2020) 
568

569

570

571

572

573

574

575

576

577

578

579

580

581

582

583

584

585

586

587

588

589

590

591

592

593

594

595

596

597

598

599

600

601

602

603

604

605

606

Ibrahim B, R C-MM, Alistair G, and J BT. 2020. Xylem cavitation isolates leaky flowers during water stress in pyrethrum. The New phytologist.

Jia D, Jiang Q, Nocker Sv, Gong X, and Ma F. 2019. An apple ( Malus domestica) NAC transcription factor enhances drought tolerance in transgenic apple plants. Plant Physiology and Biochemistry 139.

Jinxiang H, Hui Y, Xiaohua L, Zhaopu L, and Zed R. 2016. Pepino (Solanum muricatum) planting increased diversity and abundance of bacterial communities in karst area. Scientific reports 6.

Kazuo N, Hironori T, Junya M, Kazuo S, and Kazuko Y-S. 2012. NAC transcription factors in plant abiotic stress responses. Biochimica et biophysica acta 1819.

Kim S-G, Kim S-Y, and Park C-M. 2007. A membrane-associated NAC transcription factor regulates salt-responsive flowering via FLOWERING LOCUS T in Arabidopsis. Planta 226.

Kola O. 2010. Physical and chemical characteristics of the ripe pepino (Solanum muricatum) fruit grown in Turkey. Journal of Food, Agriculture, and Environment 8:168-171.

Kola O, Simsek M, Duran H, and Bozkir H. 2015. HPLC Determination of Carotenoid, Organic Acid, and Sugar Content in Pepino ( Solanum muricatum ) Fruit During the Ripening Period. Chemistry of Natural Compounds 51.

Kou, Mao, Wu, Han, Liu, Jiang, and Xue. 2017. Divergent functions of SNAC4-9 and possible mechanisms for tomato adaptation to abiotic stresses. The Journal of Horticultural Science and Biotechnology 92.

Kou X, Zhao Y, Wu C, Jiang B, Zhang Z, Rathbun JR, He Y, and Xue Z. 2018. SNAC4 and SNAC9 transcription factors show contrasting effects on tomato carotenoids biosynthesis and softening. Postharvest Biology and Technology 144.

Langeroodi ARS, Osipitan OA, Radicetti E, and Mancinelli R. 2020. To what extent arbuscular mycorrhiza can protect chicory ( Cichorium intybus L.) against drought stress. Scientia Horticulturae 263.

Lin H, Jing B, Jingyu X, and Kejun Y. 2019. Novel Maize NAC Transcriptional Repressor ZmNAC071 Confers Enhanced Sensitivity to $\mathrm{ABA}$ and Osmotic Stress by Downregulating Stress-Responsive Genes in Transgenic Arabidopsis. Journal of agricultural and food chemistry 67.

Mao H, Wang H, Liu S, Li Z, Yang X, Yan J, Li J, Tran L-SP, and Qin F. 2016. A transposable element in a NAC gene is associated with drought tolerance in maize seedlings. 第七届国际作物科学大会. 中国北京. p 1.

Pei H, Ma N, Tian J, Luo J, Chen J, Li J, Zheng Y, Chen X, Fei Z, and Gao J. 2013. An NAC Transcription Factor Controls Ethylene-Regulated Cell Expansion in Flower Petals. Plant Physiology 163.

Prohens J, Ruiz J, and Nuez F. 2002. Vegetable crop diversification in areas affected by salinity: the case of pepino (solanum muricatum). XXVI International Horticultural Congress: Environmental Stress and Horticulture Crops 618. p 267-273.

Qinqin H, Junhong Z, Hanxia L, Zhidan L, Khurram Z, Bo O, Taotao W, and Zhibiao Y. 2012. Identification and expression pattern of one stress-responsive NAC gene from Solanum lycopersicum. Molecular biology reports 39.

Rakhi C, and Swarnendu R. 2019. Evaluation of the diversity and phylogenetic implications of NAC transcription factor members of four reference species from the different embryophytic plant groups. Physiology and molecular biology of plants : an international journal of functional plant biology 25.

Schafleitner R, Kumar S, Lin C-y, Hegde SG, and Ebert A. 2013. The okra ( Abelmoschus esculentus ) transcriptome as a source for gene sequence information and molecular markers for diversity analysis. Gene 517.

Peer) reviewing PDF | (2020:06:49879:3:0:NEW 23 Dec 2020) 
607 Shathish K, and Guruvayoorappan C. 2014. Solanum muricatum Ait. Inhibits inflammation and cancer by modulating

608

609

610

611

612

613

614

615

616

617

618

619

620

621

622

623

624

625

626

627

628

629

630

631

632

633

634

635

636

637

638

639

640

641

642

643

644 the immune system. Journal of Cancer Research and Therapeutics 10.

Shathish K, Sakthivel KM, and Guruvayoorappan C. 2015. Protective Effect of Solanum muricatum on Tumor Metastasis by Regulating Inflammatory Mediators and Nuclear Factor-Kappa B Subunits. Journal of Environmental Pathology, Toxicology and Oncology 34.

Shen S, Zhang Q, Shi Y, Sun Z, Zhang Q, Hou S, Wu R, Jiang L, Zhao X, and Guo Y. 2019. Genome-Wide Analysis of the NAC Domain Transcription Factor Gene Family in Theobroma cacao. Genes 11.

Shim JS, Oh N, Chung PJ, Kim YS, Choi YD, Choi YD, and Kim J-K. 2018. Overexpression of OsNAC14 Improves Drought Tolerance in Rice. Frontiers in plant science 9.

Shuangyi Z, Yixi C, Lingling Z, Chenqi L, Jingyun Y, Tongtong L, Weiyao Y, Shengnan Z, Hongyan S, and Lei W. 2020. A novel NAC transcription factor, MdNAC42, regulates anthocyanin accumulation in red-fleshed apple by interacting with MdMYB10. Tree physiology.

Singh AK, Sharma V, Pal AK, Acharya V, and Ahuja PS. 2013. Genome-Wide Organization and Expression Profiling of the NAC Transcription Factor Family in Potato ( Solanum tuberosum L.). DNA Research 20.

Soler M, Verdaguer R, Fernández-Piñán S, Company-Arumí D, Boher P, Góngora-Castillo E, Valls M, Anticó E, Molinas M, Serra O, and Figueras M. 2020. Silencing against the conserved NAC domain of the potato StNAC103 reveals new NAC candidates to repress the suberin associated waxes in phellem. Plant Science 291.

Sudhir K, Glen S, Michael L, Christina K, and Koichiro T. 2018. MEGA X: Molecular Evolutionary Genetics Analysis across Computing Platforms. Molecular biology and evolution 35.

Sun D, Zhang X, Zhang Q, Ji X, Jia Y, Wang H, Niu L, and Zhang Y. 2019a. Comparative transcriptome profiling uncovers a Lilium regale NAC transcription factor, LrNAC35, contributing to defence response against cucumber mosaic virus and tobacco mosaic virus. Molecular Plant Pathology 20.

Sun Q, Jiang S, Zhang T, Xu H, Fang H, Zhang J, Su M, Wang Y, Zhang Z, Wang N, and Chen X. 2019b. Apple NAC transcription factor MdNAC52 regulates biosynthesis of anthocyanin and proanthocyanidin through MdMYB9 and MdMYB11. Plant Science 289.

Swati P, Pankaj SP, S SP, and Manoj P. 2012. NAC proteins: regulation and role in stress tolerance. Trends in plant science 17.

Tadashi I, and Ken T. 2019. Insect and mite pests of pepino ( Solanum muricatum Ait.) in Japan. Biodiversity data journal 7.

Tweneboah S, and Oh S-K. 2017. Biological roles of NAC transcription factors in the regulation of biotic and abiotic stress responses in solanaceous crops. Journal of Plant Biotechnology 44:1-11.

Wang G, Zhang S, Ma X, Wang Y, Kong F, and Meng Q. 2016. A stress-associated NAC transcription factor ( SINAC35 ) from tomato plays a positive role in biotic and abiotic stresses. Physiologia Plantarum 158.

Wang S, Zhou G, Huang X, Hu J, Wang B, Lin C, Li X, Jia Y, and Wang A. 2017. Transcriptome analysis of non-heading Chinese cabbage under heat stress by RNA-seq and marker identification. Euphytica 213.

Wang Y, Cao S, Guan C, Kong X, Wang Y, Cui Y, Liu B, Zhou Y, and Zhang Y. 2020. Overexpressing the NAC transcription factor LpNAC13 from Lilium pumilum in tobacco negatively regulates the drought response and positively regulates the salt response. Plant Physiology and Biochemistry 149.

Peer) reviewing PDF | (2020:06:49879:3:0:NEW 23 Dec 2020) 
645 Wei-Li G, Shu-Bin W, Ru-Gang C, Bi-Hua C, Xiao-Hua D, Yan-Xu Y, Zhen-Hui G, and Yu-Yuan Z. 2015. 646 Characterization and expression profile of CaNAC2 pepper gene. Frontiers in plant science 6.

647 Wei L, Xiaoxu L, Jiangtao C, Zenglin Z, Weifeng W, and Yongfeng G. 2018. NAC Family Transcription Factors in 648 Tobacco and Their Potential Role in Regulating Leaf Senescence. Frontiers in plant science 9.

649 Weiping D, C SJ, Shubin W, Jinbing L, Baogui P, Guangjun G, Wei G, and Ali DMHS. 2018. Genome-Wide Analyses of the NAC Transcription Factor Gene Family in Pepper (Capsicum annuum L.): Chromosome Location, Phylogeny, Structure, Expression Patterns, Cis-Elements in the Promoter, and Interaction Network. International journal of molecular sciences 19.

Xi-Man K, Qian Z, Xin Z, Bao-Dong W, and Shu-Juan J. 2020. Transcription factor CaNAC1 regulates low-temperatureinduced phospholipid degradation in green bell pepper. Journal of experimental botany 71.

Xin-Jian H, Rui-Ling M, Wan-Hong C, Zhi-Gang Z, Jin-Song Z, and Shou-Yi C. 2005. AtNAC2, a transcription factor downstream of ethylene and auxin signaling pathways, is involved in salt stress response and lateral root development. The Plant journal : for cell and molecular biology 44.

Xin G, Liangyi Z, Xiaofei S, Zekun L, Bingjie G, Jinxuan Y, Shaoling Z, Shutian T, and Xiaosan H. 2019. Genome-wide analyses and expression patterns under abiotic stress of NAC transcription factors in white pear (Pyrus bretschneideri). BMC plant biology 19.

Xinyue P, Min X, Meiyan R, Dina N, Yaqi W, and Huiqin G. 2019. Ammopiptanthus mongolicus stress-responsive NAC gene enhances the tolerance of transgenic Arabidopsis thaliana to drought and cold stresses. Genetics and molecular biology 42.

Xuemeng S, Kebin Y, Xiurong X, Chenglei Z, and Zhimin G. 2019. Genome-Wide Investigation of the NAC Gene Family and Its Potential Association with the Secondary Cell Wall in Moso Bamboo. Biomolecules 9.

Xuemin M, Youjun Z, Veronika T, Gang-Ping X, R FA, Bernd M-R, and Salma B. 2018. The NAC Transcription Factor SINAP2 Regulates Leaf Senescence and Fruit Yield in Tomato. Plant Physiology.

Yong Y, Zhang Y, and Lyu Y. 2019. A Stress-Responsive NAC Transcription Factor from Tiger Lily (LINAC2) Interacts with LIDREB1 and L1ZHFD4 and Enhances Various Abiotic Stress Tolerance in Arabidopsis. International journal of molecular sciences 20.

Yongil Y, Geun YC, William R, A WK, M CC, E GL, S JS, Xiaohan Y, Yunqiao P, J RA, A TG, and Jin-Gui C. 2019. PdWND3A, a wood-associated NAC domain-containing protein, affects lignin biosynthesis and composition in Populus. BMC plant biology 19.

Yuan X, Wang H, Cai J, Bi Y, Li D, and Song F. 2019. Rice NAC transcription factor ONAC066 functions as a positive regulator of drought and oxidative stress response. BioMed Central 19.

Yujie F, Jun Y, Kabin X, Weibo X, and Lizhong X. 2008. Systematic sequence analysis and identification of tissue-specific or stress-responsive genes of NAC transcription factor family in rice. Molecular genetics and genomics : MGG 280.

679 Yunjun Z, Jiayan S, Peng X, Rui Z, and Laigeng L. 2014. Intron-mediated alternative splicing of WOOD-ASSOCIATED 680 NAC TRANSCRIPTION FACTOR1B regulates cell wall thickening during fiber development in Populus species. Plant Physiology 164. 
682 Zamarud S, Hussain SS, Shad AG, Iqbal M, Sher KR, Arshad I, Nisar A, and Asad J. 2020. Introduction of Arabidopsis's 683 heat shock factor HsfA1d mitigates adverse effects of heat stress on potato (Solanum tuberosum L.) plant. Cell 684 stress $\&$ chaperones 25.

685 Zhang J, Li L, Huang L, Zhang M, Chen Z, Zheng Q, Zhao H, Chen X, Jiang M, and Tan M. 2019. Maize NAC-domain 686 retained splice variants act as dominant negatives to interfere with the full-length NAC counterparts. Plant $687 \quad$ Science 289.

688 Zhang Y, Li D, Wang Y, Zhou R, Wang L, Zhang Y, Yu J, Gong H, You J, and Zhang X. 2018. Genome-wide identification 689 and comprehensive analysis of the NAC transcription factor family in Sesamum indicum. PloS one 13.

6901.

691

692

693

694

695

696

697

698

699

700

701

702

703

704

705

706 


\section{Figure 1}

The position of the 44 conserved domains of SmNAC protein on the amino acid chain.

The position of the 44 conserved domains of SmNAC protein on the amino acid chain. The rectangles of different colors represent different motifs and their positions on each of the NAC sequences. The correspondence between the rectangles of each color and motif is shown in the upper right corner of the figure. The horizontal axis at the bottom of the figure is the reference axis of the length of the amino acid chain.

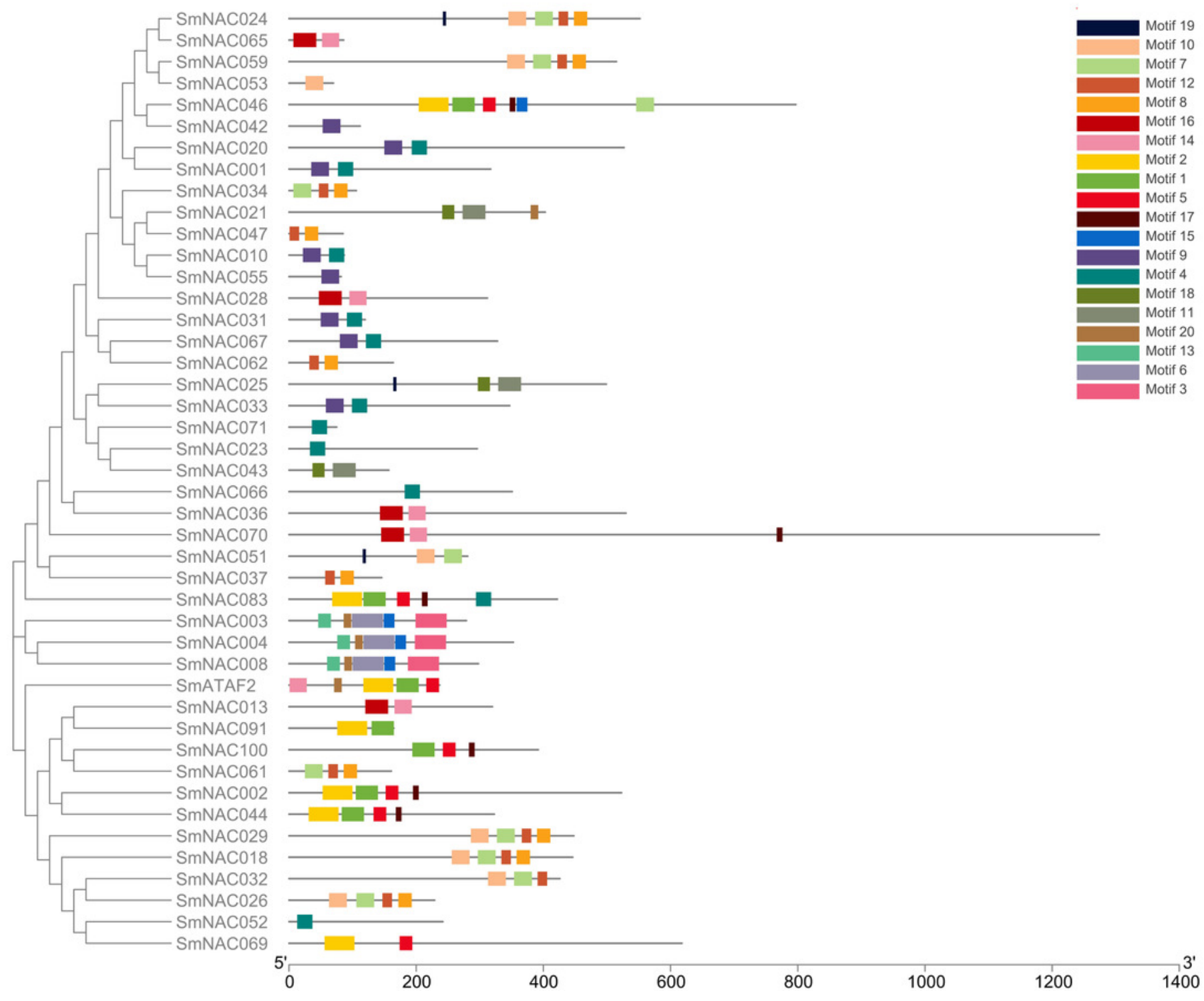




\section{Figure 2}

The 20 conserved motifs (Motifs 1 to 20) identified in Solanum muricatum NAC proteins.

The 20 conserved motifs (Motifs 1 to 20) identified in Solanum muricatum NAC proteins. The length, degree of conservation, and the sharing or substitution of every amino acid in each of the motifs are displayed. Each motif had a corresponding coordinate axis, the abscissa represents the corresponding position of each amino acid on the motif, and the ordinate represents sections. 
Motif1

A Motif2

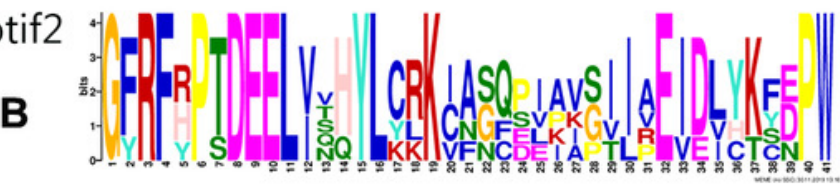

Motif11

Motif12

L

Motif3 it || Motif13

C

M

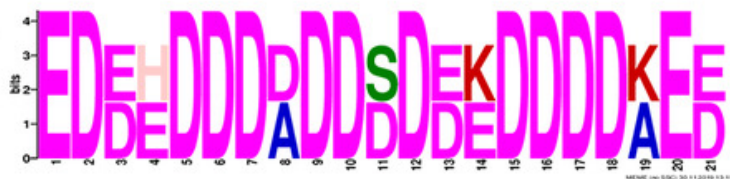

Motif4

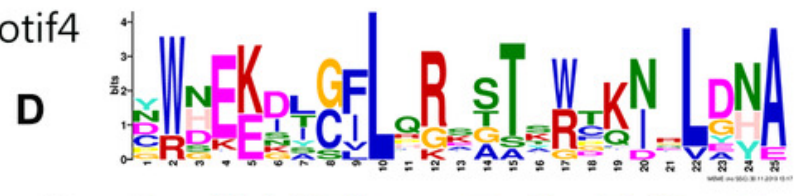

Motif5

Motif6

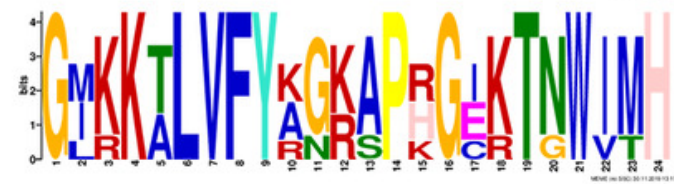 \\ E}

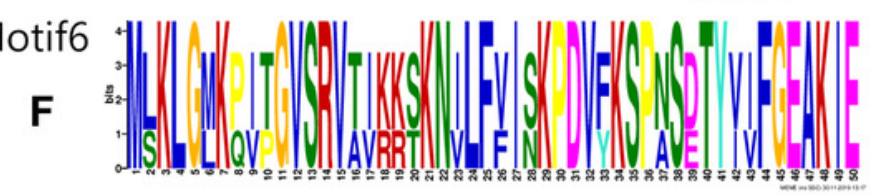

Motif7

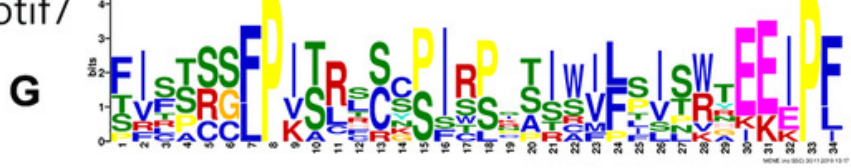

Motif8

H

Motif9

I

Motif10

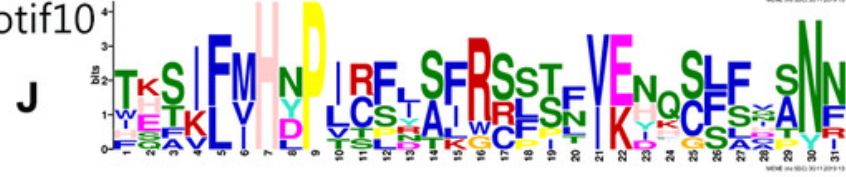

Motif14

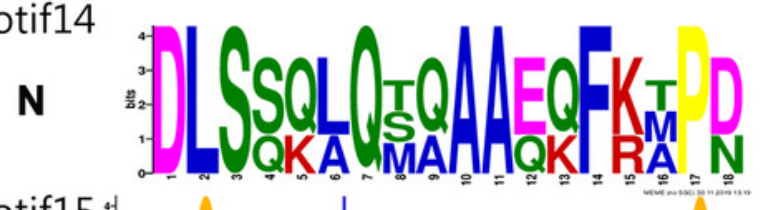

Motif15 0 .

Motif16

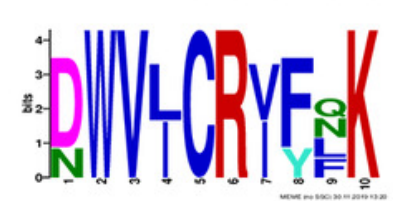

Motif17

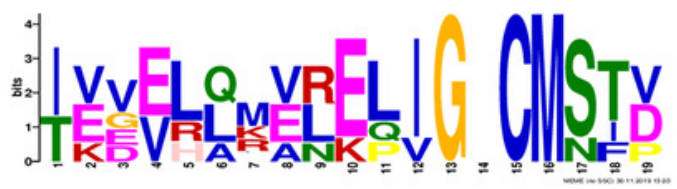

Motif18

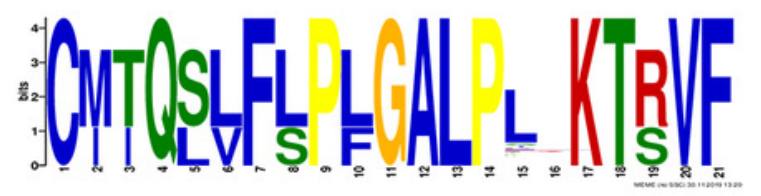

Motif19

$\mathbf{S}$

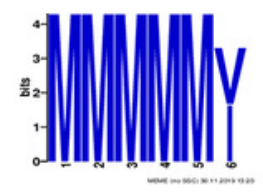

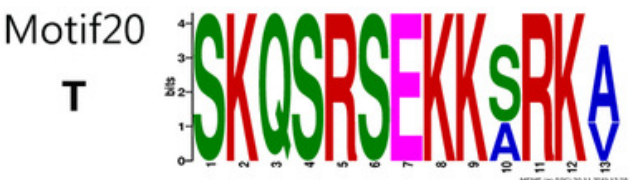




\section{Figure 3}

Unrooted phylogenetic tree showing the relationship between the NAC family members of Arabidopsis, pepper, tomato, potato, and Solanum muricatum.

Unrooted phylogenetic tree showing the relationship between the NAC family members of Arabidopsis, pepper, tomato, potato, and Solanum muricatum. Each species is represented by a background color as shown in the upper left corner of the figure. Classes of different colors represented different subfamilies of Solanum muricatum NAC. 


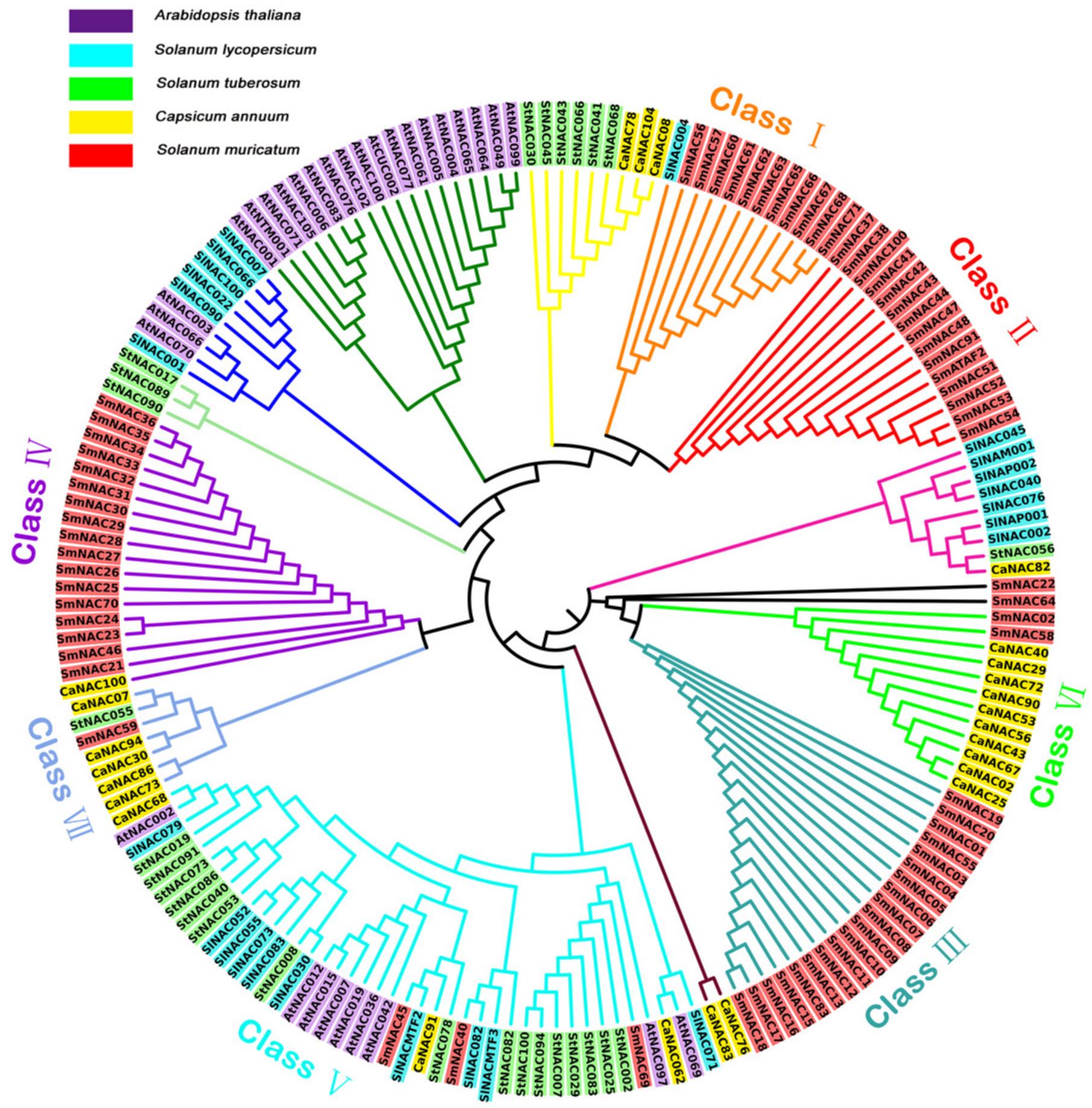




\section{Figure 4}

Changes in plant shape of Solanum muricatum seedlings with 7 weeks old after drought stress

This figure shows the changes in plant shape of Solanum muricatum seedlings with 7 weeks old after drought stress. The drought stress experiment was conducted in an incubator at $24^{\circ} \mathrm{C}$ with $70 \%$ relative air humidity, and $16 \mathrm{~h} / \mathrm{d}$ light. The red gradient deepening process indicated the passage of drought stress time, and the green gradient deepening process indicated the increase of PEG solution concentration. The photos show the morphological changes of Solanum muricatum plants at various sampling time points ( $4 \mathrm{~h}, 8 \mathrm{~h}, 12 \mathrm{~h}, 18 \mathrm{~h}$, $24 \mathrm{~h}$, and $32 \mathrm{~h}$ ) and in the presence of PEG at 3 different concentrations (5\%,10\%, and $15 \%)$.

Date of experiment:from 11:30,Wednesday,October 16th,2019 to 23:30,Thursday,October 17th,2019 Relative air humidity:70\%

Air temperature: $24 \mathrm{C}$

Illumination hours:16h

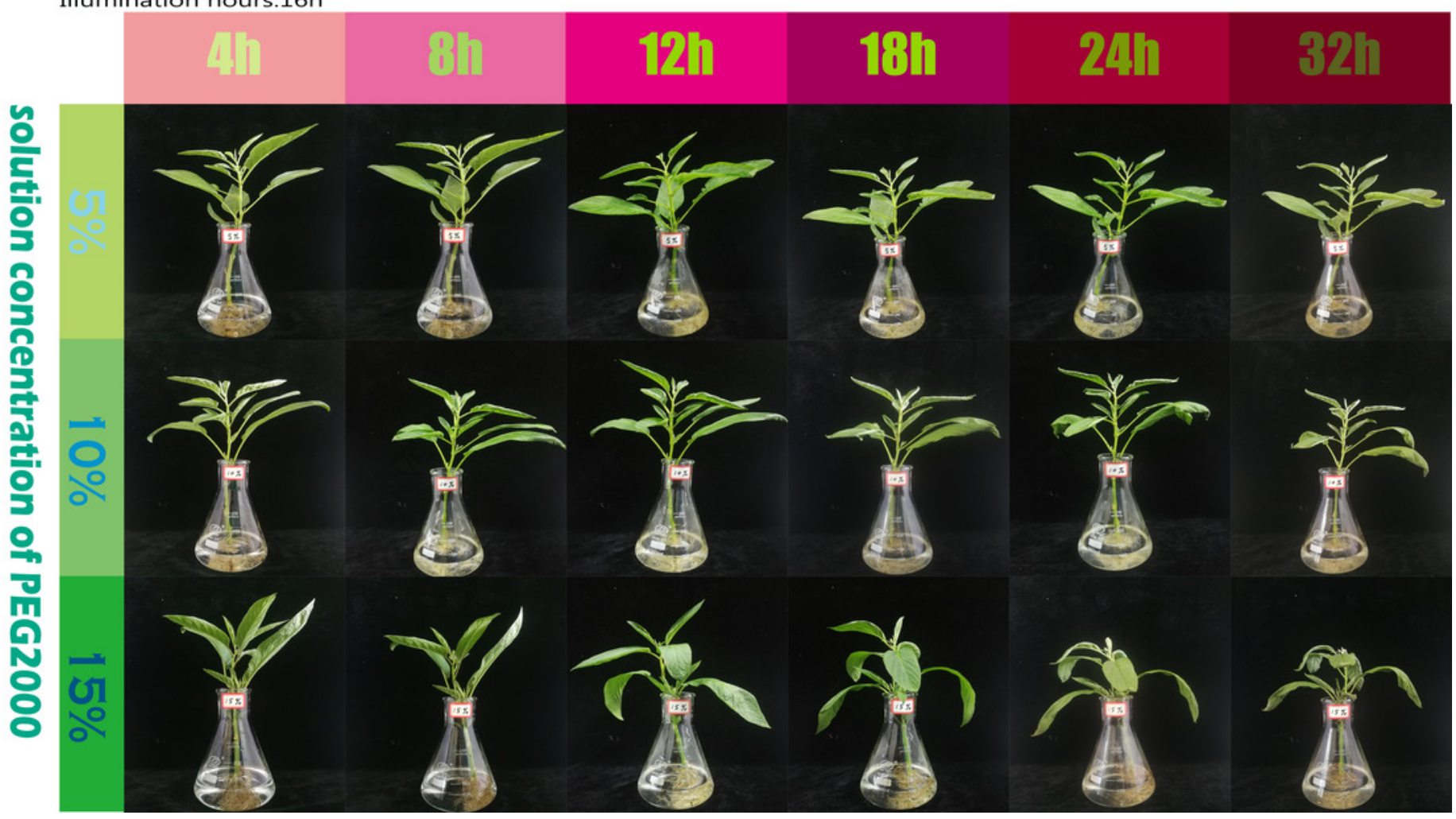




\section{Figure 5}

Heat map

The Heat map shows the expression of Solanum muricatum NAC genes in four tissues of root, stem, leaf, and flower under drought stress. A positive value meant higher than the average level, 0 was the average level, and a negative value represents below-average levels. 


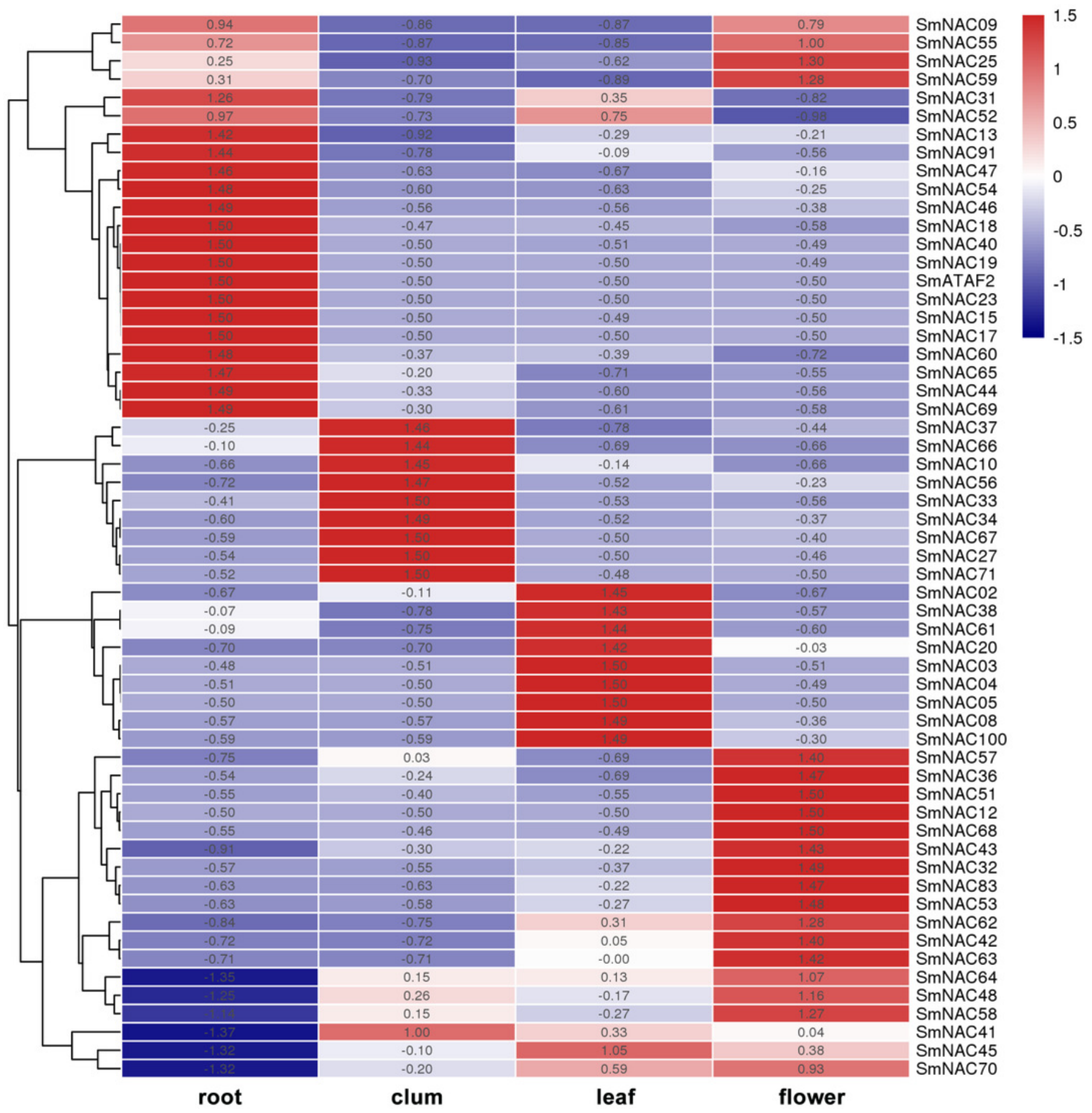




\section{Figure 6}

Changes in the expression of the selected 7 Solanum muricatum NAC genes over time under different degrees of drought stress

The histogram shows the changes in the expression of the selected 7 Solanum muricatum NAC genes over time under different degrees of drought stress. Three kinds of PEG solutions with $5 \%, 10 \%$, and $15 \%$ concentrations were used to stress the Solanum muricatum plants respectively. The leaves of the plants were taken after $0 \mathrm{~h}, 4 \mathrm{~h}, 8 \mathrm{~h}, 12 \mathrm{~h}, 18 \mathrm{~h}, 24 \mathrm{~h}$, and $32 \mathrm{~h}$, and the expressions of Solanum muricatum NAC gene were detected by qPCR. The 2- $\triangle \triangle C T$ method was used to calculate the relative expression of the target genes. The abscissa represents each sampling time point of the experiment (4h, 8h, 12h, 18h, 24h, and 32h). The ordinate indicates the relative expression levels. White, gray, and black represent $5 \%, 10 \%$, and $15 \%$ PEG stress experimental groups, respectively. 

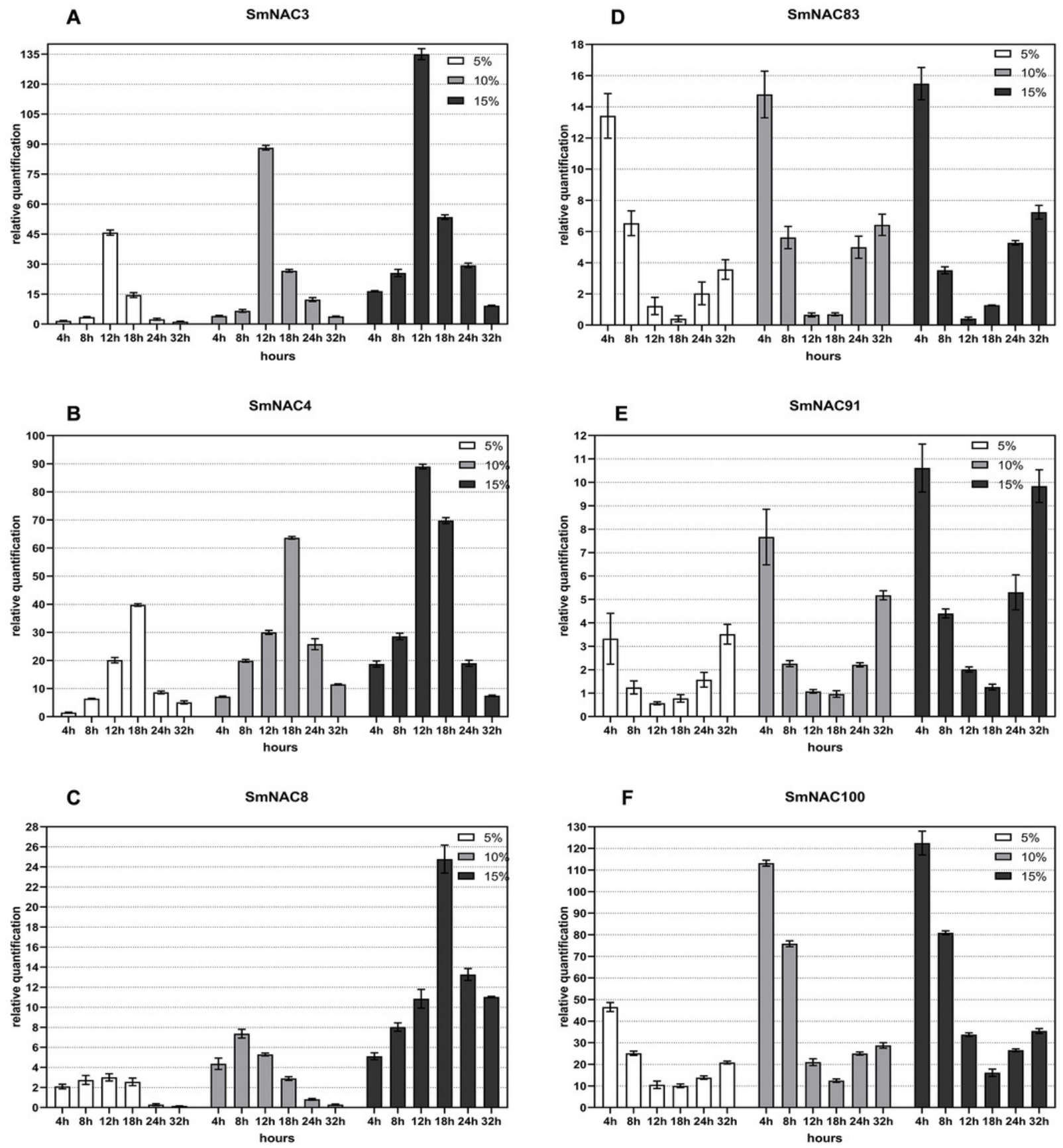

G

SmNAC69

$4 \mathrm{~h} 8 \mathrm{~h} 12 \mathrm{~h} 18 \mathrm{~h} \mathrm{24h} 32 \mathrm{~h} \quad 4 \mathrm{~h} 8 \mathrm{~h} 12 \mathrm{~h} 18 \mathrm{~h} 24 \mathrm{~h} 32 \mathrm{~h} \quad 4 \mathrm{~h}$ 8h $12 \mathrm{~h} 18 \mathrm{~h} 24 \mathrm{~h} 32 \mathrm{~h}$

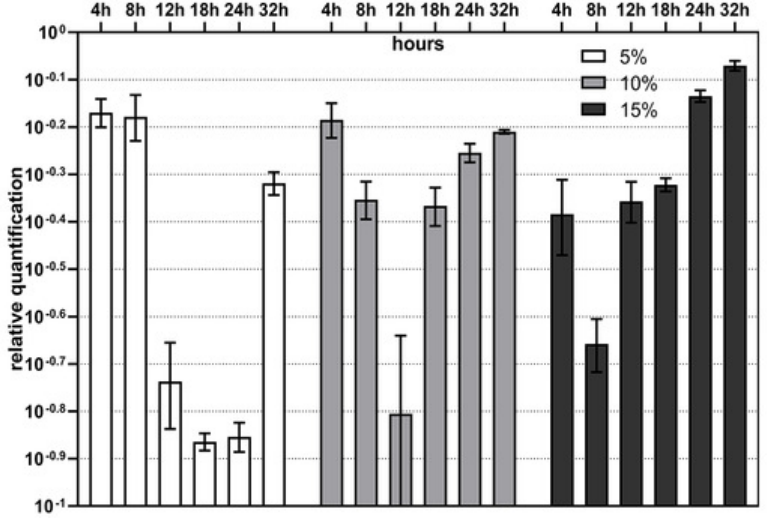




\section{Figure 7}

Multiple sequence alignments of four groups of NAC genes.

Multiple sequence alignments of four groups of NAC genes. The Zappo Colours color scheme (http://www.jalview.org/version118/documentation.html\#zappo) was used to present the amino acid sequence. Note: (Conservation: Conservation of total alignment of less than $25 \%$ gaps. Quality: Alignment Quality based on Blosum62 scores. Consensus: PID ( http://www.jalview.org/version118/documentation.html\#pid ). Occupancy: Number of aligned positions.)

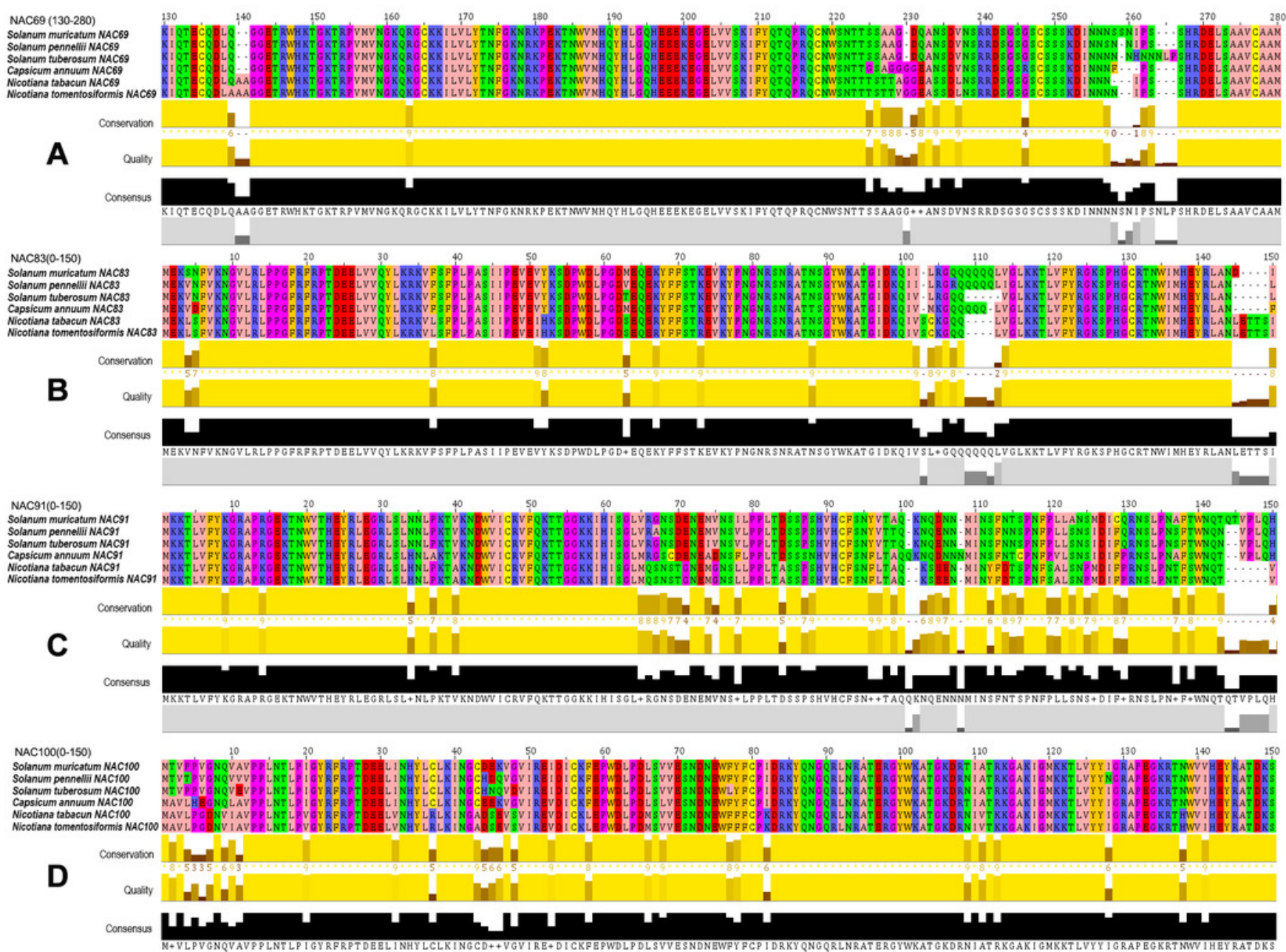




\section{Table 1 (on next page)}

PCR primer information table

The information of upstream and downstream primers used for the screening of 7 SmNAC genes and the annealing temperature set during qPCR assay. 


\begin{tabular}{crc}
\hline Primer name & Sequence (5'-3') & Annealing temperature \\
\hline SmNAC03-1-F & GGTTAGGTCCATAATAGCA & $52^{\circ} \mathrm{C}$ \\
SmNAC03-1-R & AGCCAATGTCAACAAGAG & \\
SmNAC04-1-F & CTGCCACTACCATCACCAAG & $57^{\circ} \mathrm{C}$ \\
SmNAC04-1-R & TTCTAGCCGCTCATCTCG & \\
SmNAC08-1-F & TCAGAATCGTCGGCGTCA & $58^{\circ} \mathrm{C}$ \\
SmNAC08-1-R & TCCTCCCGTTCCATCACA & \\
SmNAC83-1-F & TTGTAAACTTCAACCTCC & $51^{\circ} \mathrm{C}$ \\
SmNAC83-1-R & TGTATTAAGATTACCACCAG & \\
SmNAC100-1-F & CAAGCCCTGAAATGTGAA & $58^{\circ} \mathrm{C}$ \\
SmNAC100-1-R & CAGTAAAGAATGATTGGGTGAT & \\
SmNAC91-1-F & GTATCCTCGTTCTGTTGC & $58^{\circ} \mathrm{C}$ \\
SmNAC91-1-R & GCCTGATTTGTCTGTTGT & \\
SmNAC69-1-F & TGCAGACCTTGCTCCTAG & $56^{\circ} \mathrm{C}$ \\
SmNAC69-1-R & ATAACTCCGCCTCCACTC & \\
APRT121-R & GAACCGGAGCAGGTGAAGAA & $60{ }^{\circ} \mathrm{C}$ \\
APRT121-D & GAAGCAATCCCAGCGATACG & \\
\hline
\end{tabular}




\section{Table 2 (on next page)}

The analysis of physical and chemical properties of SmNAC genes

Structural, physical, and chemical properties of the 71 Solanum muricatum NAC gene family proteins, including the length of CDNA, amino acid length, protein molecular mass, isoelectric point, hydrophilicity, and aliphatic amino acid index of each SmNAC. 


\begin{tabular}{|c|c|c|c|c|c|c|}
\hline Gene name & $\begin{array}{l}\text { Number of } \\
\text { amino acid }\end{array}$ & $\begin{array}{c}\text { Length of } \\
\text { cDNA }\end{array}$ & $\begin{array}{c}\text { Theoretical } \\
\text { pI }\end{array}$ & $\begin{array}{c}\text { Molecular } \\
\text { weight }\end{array}$ & Aliphatic index & $\begin{array}{c}\text { Grand average of } \\
\text { hydropathicity } \\
\text { (GRAVY) }\end{array}$ \\
\hline SmNAC001 & 281 & 952 & 9.89 & 33076.93 & 74.91 & -0.556 \\
\hline SmNAC055 & 72 & 246 & 9.89 & 8623.95 & 81.25 & -0.804 \\
\hline SmNAC003 & 269 & 839 & 4.84 & 29167.80 & 81.97 & -0.314 \\
\hline SmNAC004 & 349 & 1060 & 5.00 & 38489.85 & 91.35 & -0.275 \\
\hline SmNAC005 & 242 & 755 & 9.61 & 26710.34 & 92.73 & 0.293 \\
\hline SmNAC006 & 250 & 821 & 9.15 & 29081.58 & 87.76 & -0.346 \\
\hline SmNAC007 & 202 & 637 & 9.84 & 22121.79 & 98.91 & 0.371 \\
\hline SmNAC008 & 292 & 896 & 5.17 & 32702.08 & 78.12 & -0.454 \\
\hline SmNAC009 & 174 & 547 & 9.58 & 20602.20 & 139.48 & 0.732 \\
\hline SmNAC010 & 75 & 263 & 9.28 & 9084.77 & 78.00 & -0.12 \\
\hline SmNAC011 & 78 & 252 & 9.93 & 9676.84 & 111.03 & 0.273 \\
\hline SmNAC012 & 439 & 1393 & 6.65 & 51160.53 & 81.16 & -0.319 \\
\hline SmNAC013 & 303 & 962 & 9.98 & 35144.76 & 102.54 & 0.391 \\
\hline SmNAC083 & 417 & 1267 & 9.51 & 48606.85 & 79.88 & -0.456 \\
\hline SmNAC015 & 383 & 1228 & 8.31 & 44992.07 & 103.79 & 0.173 \\
\hline SmNAC016 & 89 & 274 & 10.58 & 9763.43 & 38.54 & -1.311 \\
\hline SmNAC017 & 86 & 278 & 10.02 & 9683.25 & 106.51 & 0.294 \\
\hline SmNAC018 & 415 & 1338 & 9.81 & 48541.71 & 113.18 & 0.342 \\
\hline SmNAC019 & 190 & 603 & 9.15 & 22632.29 & 108.26 & 0.038 \\
\hline SmNAC020 & 488 & 1583 & 10.32 & 57480.91 & 87.21 & -0.384 \\
\hline SmNAC021 & 386 & 1209 & 9.92 & 43804.80 & 110.57 & 0.295 \\
\hline SmNAC022 & 834 & 2632 & 10.38 & 98282.25 & 99.46 & -0.017 \\
\hline SmNAC023 & 264 & 889 & 9.63 & 30651.24 & 89.43 & -0.209 \\
\hline SmNAC024 & 510 & 1657 & 9.77 & 59479.00 & 110.61 & 0.281 \\
\hline SmNAC025 & 467 & 1499 & 9.58 & 53875.50 & 103.45 & 0.204 \\
\hline SmNAC026 & 216 & 687 & 9.27 & 25484.21 & 107.87 & 0.582 \\
\hline SmNAC027 & 159 & 547 & 9.85 & 18850.57 & 87.04 & -0.525 \\
\hline SmNAC028 & 299 & 937 & 9.97 & 34173.24 & 121.91 & 0.426 \\
\hline SmNAC029 & 432 & 1345 & 10.17 & 50313.25 & 100.09 & 0.027 \\
\hline SmNAC030 & 186 & 592 & 9.58 & 21963.10 & 129.84 & 0.433 \\
\hline SmNAC031 & 109 & 361 & 9.37 & 12902.56 & 58.17 & -1.08 \\
\hline SmNAC032 & 405 & 1279 & 10.37 & 47851.51 & 101.98 & 0.081 \\
\hline SmNAC033 & 297 & 1043 & 10.58 & 34504.44 & 66.33 & -0.799 \\
\hline
\end{tabular}




\begin{tabular}{|c|c|c|c|c|c|c|}
\hline SmNAC034 & 105 & 320 & 8.90 & 12591.10 & 130.76 & 0.533 \\
\hline SmNAC035 & 96 & 292 & 9.58 & 11396.40 & 74.06 & -0.708 \\
\hline SmNAC036 & 497 & 1591 & 10.46 & 57968.21 & 105.92 & 0.056 \\
\hline SmNAC037 & 140 & 440 & 10.37 & 16809.58 & 84.21 & -0.711 \\
\hline SmNAC038 & 139 & 425 & 9.62 & 15597.49 & 119.78 & 0.489 \\
\hline SmNAC100 & 379 & 1177 & 10.04 & 44033.32 & 87.18 & -0.199 \\
\hline SmNAC040 & 517 & 1583 & 10.03 & 61149.56 & 102.34 & 0.227 \\
\hline SmNAC041 & 258 & 824 & 10.83 & 30624.21 & 93.64 & -0.107 \\
\hline SmNAC042 & 102 & 338 & 5.41 & 11987.43 & 63.04 & -0.689 \\
\hline SmNAC043 & 151 & 471 & 9.50 & 16870.03 & 116.16 & 0.604 \\
\hline SmNAC044 & 319 & 969 & 7.22 & 37036.11 & 76.39 & -0.51 \\
\hline SmNAC045 & 466 & 1550 & 8.99 & 53488.19 & 88.22 & 0.001 \\
\hline SmNAC046 & 775 & 2393 & 9.16 & 88790.28 & 71.07 & -0.655 \\
\hline SmNAC047 & 82 & 255 & 10.10 & 9988.00 & 124.76 & 0.157 \\
\hline SmNAC048 & 109 & 357 & 5.71 & 12878.40 & 52.75 & -0.585 \\
\hline SmNAC091 & 164 & 495 & 6.85 & 18780.42 & 77.74 & -0.329 \\
\hline SmATAF2 & 233 & 713 & 9.62 & 26838.87 & 74.12 & -0.551 \\
\hline SmNAC051 & 269 & 844 & 10.41 & 31395.21 & 106.80 & 0.205 \\
\hline SmNAC052 & 206 & 728 & 9.36 & 24289.65 & 73.30 & -0.65 \\
\hline SmNAC053 & 69 & 211 & 8.06 & 7808.08 & 98.70 & 0.472 \\
\hline SmNAC054 & 550 & 1722 & 10.20 & 63209.67 & 119.78 & 0.486 \\
\hline SmNAC002 & 516 & 1569 & 9.29 & 59616.37 & 82.95 & -0.169 \\
\hline SmNAC056 & 430 & 1315 & 9.34 & 47617.09 & 88.67 & -0.193 \\
\hline SmNAC057 & 289 & 879 & 6.44 & 33405.21 & 71.19 & -0.323 \\
\hline SmNAC058 & 876 & 2748 & 9.40 & 101501.11 & 87.76 & -0.366 \\
\hline SmNAC059 & 496 & 1546 & 9.87 & 58173.58 & 91.57 & -0.034 \\
\hline SmNAC060 & 428 & 1384 & 9.36 & 49835.10 & 88.57 & -0.239 \\
\hline SmNAC061 & 151 & 485 & 10.41 & 17769.13 & 94.24 & 0.196 \\
\hline SmNAC062 & 161 & 492 & 10.23 & 19508.97 & 85.90 & -0.054 \\
\hline SmNAC063 & 64 & 211 & 6.02 & 7730.94 & 109.53 & 0.283 \\
\hline SmNAC064 & 805 & 2555 & 9.06 & 92552.60 & 74.48 & -0.531 \\
\hline SmNAC065 & 81 & 258 & 9.60 & 9069.87 & 108.02 & -0.007 \\
\hline SmNAC066 & 306 & 1055 & 9.92 & 36121.10 & 98.50 & 0.241 \\
\hline SmNAC067 & 297 & 985 & 9.88 & 34929.32 & 85.69 & -0.179 \\
\hline SmNAC068 & 85 & 292 & 4.67 & 10191.52 & 104.35 & -0.466 \\
\hline SmNAC069 & 617 & 1855 & 8.72 & 70549.22 & 69.71 & -0.649 \\
\hline
\end{tabular}




\begin{tabular}{lcccccc} 
SmNAC070 & 1201 & 3823 & 9.75 & 139153.20 & 107.37 & 0.33 \\
SmNAC071 & 68 & 225 & 6.90 & 8011.07 & 81.76 & -0.475 \\
\hline
\end{tabular}

